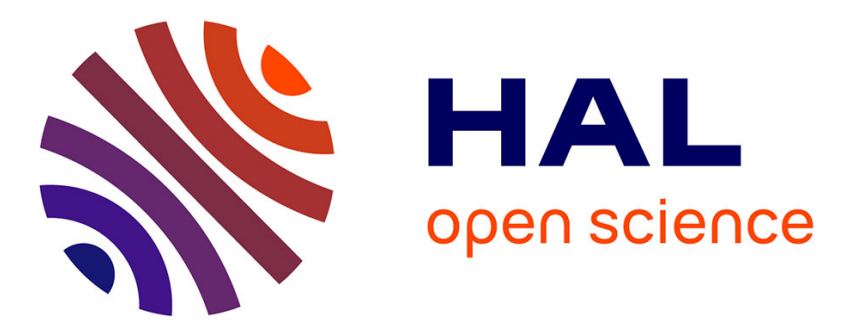

\title{
On a Multiscale Computational Strategy with Time and Space Homogenization for Structural Mechanics
}

\author{
Pierre Ladevèze, Anthony Nouy
}

\section{To cite this version:}

Pierre Ladevèze, Anthony Nouy. On a Multiscale Computational Strategy with Time and Space Homogenization for Structural Mechanics. Computer Methods in Applied Mechanics and Engineering, 2003, 192 (28-30), pp.3061-3087. 10.1016/S0045-7825(03)00341-4 . hal-00366648

\section{HAL Id: hal-00366648 https://hal.science/hal-00366648}

Submitted on 9 Mar 2009

HAL is a multi-disciplinary open access archive for the deposit and dissemination of scientific research documents, whether they are published or not. The documents may come from teaching and research institutions in France or abroad, or from public or private research centers.
L'archive ouverte pluridisciplinaire HAL, est destinée au dépôt et à la diffusion de documents scientifiques de niveau recherche, publiés ou non, émanant des établissements d'enseignement et de recherche français ou étrangers, des laboratoires publics ou privés. 


\title{
On a Multiscale Computational Strategy with Time and Space Homogenization for Structural Mechanics
}

\author{
Pierre Ladevèze, Anthony Nouy \\ LMT-Cachan (ENS Cachan / CNRS / Université Paris VI) \\ 61 Avenue du Président Wilson, F-94235 Cachan CEDEX, France
}

\begin{abstract}
A new multiscale computational strategy was recently proposed for the analysis of structures described both on a fine space scale and a fine time scale. This strategy, which involves homogenization in space as well as in time, could replace in several domains of application the standard homogenization techniques, which are generally limited to the space domain. It is an iterative strategy which calls for the resolution of problems on both a micro (fine) scale and a macro (homogenized) scale. In this paper, we review the bases of this approach and present improved approximation techniques to solve the micro and macro problems.
\end{abstract}

Key words: Homogenization, Multiscale, Domain decomposition

\section{Introduction}

In the structural mechanics field, one can observe a growing interest in the multiscale analysis of structures with complex microstructural geometry and behavior. When an accurate solution is required, calculations must be performed on a finely discretized model of the structure (on the micro level). Since its components often have very different mechanical characteristics, the resulting structure is highly heterogeneous and the local solution involves phenomena with a short length of variation both in space and in time. This type of situation leads to problems with very large numbers of degrees of freedom whose calculation costs are generally prohibitive if one uses classical FE codes. Our objective is to reduce the calculation costs drastically while, at the same time, trying to improve robustness.

One possible strategy, especially for linear problems, consists of applying the theory of periodic media homogenization initiated by Sanchez-Palencia [20]. 
Further developments of related computational approaches can be found in $[4,8,9,11,19,21,22]$. The macro problem yields effective values of the unknowns; then, the micro solution must be calculated locally in terms of the macro solution. Besides periodicity, the fundamental assumption is that the ratio of the small-scale length to the large-scale length must be small. Boundary zones, in which the material cannot be homogenized, require special treatment. Moreover, this theory is not directly applicable to time-dependent nonlinear problems.

Relatively few works have been devoted to multi-time-scale computational strategies. The so-called multi-time-step methods [1,12] and time-decomposed parallel time-integrators [7] deal with different time discretizations and integration schemes. Local enrichment functions were introduced in [3]. In multiphysics problems, coupling between time grids may be envisaged. This type of problem was solved in [5] by introducing "micro-macro projectors" between grids. None of these strategies involves a true time-homogenization technique. Such a technique seems to have been used only for periodic loading histories (see $[2,10,13,14])$.

A first answer to our challenge was to devise a new micro-macro computational strategy [15] which includes a space homogenization technique valid on the whole domain, while avoiding the drawbacks of the classical homogenization theory. This technique was extended to both space and time in $[17,18]$. The strategy is iterative and works over the entire space-time domain. Here, this strategy will be detailed for (visco)plastic materials and optional unilateral contact with or without friction, a case already introduced in [16]. More complex material behaviors could be taken into account.

The first characteristic of the method resides in a partitioning of the spacetime domain. The structure is defined as an assembly of substructures and interfaces. Each component has its own variables and its own equations. The time interval is divided into subintervals, using the discontinuous Galerkin method to handle possible discontinuities. The junction between the macro and the micro scales takes place only at the interfaces.

The second characteristic of the method is the use of the so-called LATIN method, a nonincremental iterative computational strategy applied over the entire time interval being studied [14]. At each iteration, one must solve a macro problem, defined over the entire structure and the entire time interval, along with a family of independent linear problems each defined on a composite cell and its boundary. These are "micro" problems, by contrast with the "macro" problem which corresponds to the entire structure homogenized in time as well as in space.

After reviewing the bases of the multiscale strategy with space and time ho- 
mogenization, this paper will concentrate on approximation techniques suitable for the resolution of the micro and macro problems.

A first improvement consists of introducing a third scale, which can be very useful for many composite structures. This can be viewed as an approximation of the macro problem, represented as a Cosserat-like medium. On this third scale, the representative volume is defined as an assembly of substructures (i.e composite cells).

Another improvement concerns the resolution over the space-time domain of the micro problem within cells, which can be very large if the micro description is detailed. A "radial-type approximation" [14] is introduced. This technique consists of approximating a function defined on the space-time domain by a sum of products of scalar functions of the time variable by functions of the space variable. As the iterations proceed, the functions of the space variable constructed in this manner constitute a consistent basis which can be reused for the following iterations. Moreover, when dealing with similar substructures, such as composite cells, this basis is common to all the substructures. Several numerical examples will illustrate the capabilities of the approach presented and the improvements made possible by these approximation techniques.

\section{The reference problem}

\subsection{Description of the problem}

Let us consider, under the assumptions of small perturbations and isothermal, quasi-static state, the equilibrium of a structure defined in the space domain $\Omega$. At each time $t$ of the interval $[0, T]$ being studied, this structure is subjected to volume forces $\underline{\mathrm{f}}_{d}$ and surface forces $\underline{\mathrm{F}}_{d}$ on a portion $\partial_{2} \Omega$ of the boundary. On the complementary part $\partial_{1} \Omega$, the displacement $\underline{u}_{d}$ is prescribed. All the quantities with subscript "d" are known. Displacements, strains and stresses are subject to initial conditions at $t=0$.

\subsection{Constitutive relation model with internal state variables}

The inelastic strain $\varepsilon_{p}$ is considered separately from the other internal variables, denoted $\mathbf{X}$. The conjugate variable of $\mathbf{X}$ is $\mathbf{Y}$; thus, the dissipation rate is: 


$$
\operatorname{Tr}\left[\boldsymbol{\sigma} \dot{\varepsilon}_{p}\right]-\mathbf{Y} \cdot \dot{\mathbf{X}}
$$

From the free energy $\rho \Psi\left(\varepsilon_{e}, \mathbf{X}\right)$, under usual decoupling assumptions, we have:

$$
\left\{\begin{array}{l}
\boldsymbol{\sigma}=\rho \frac{\partial \Psi}{\partial \boldsymbol{\varepsilon}_{e}}=\mathbf{K} \boldsymbol{\varepsilon}_{e} \\
\mathbf{Y}=\rho \frac{\partial \Psi}{\partial \mathbf{X}}=\boldsymbol{\Lambda} \mathbf{X}
\end{array}\right.
$$

where the Hooke's tensor $\mathbf{K}$ and the operator $\boldsymbol{\Lambda}$ are material characteristics. The advantage of such a model lies in the simple expression of the corresponding constitutive relation, which, except for (1), can be written as a differential equation:

$$
\frac{d}{d t}\left[\begin{array}{c}
\varepsilon_{p} \\
-\mathbf{X}
\end{array}\right]=\mathbf{B}\left(\left[\begin{array}{c}
\boldsymbol{\sigma} \\
\mathbf{Y}
\end{array}\right]\right), \quad \varepsilon_{p \mid t=0}=0, \quad \mathbf{X}_{\mid t=0}=0
$$

where $\mathbf{B}$ is a positive operator characterizing the material.

This formulation is "normal" in the sense that the operator $\boldsymbol{\Lambda}$ is linear, constant, symmetric and positive definite. With a change of internal variables, most material models can be described by such a normal formulation [14].

\subsection{Partitioning of the structure into substructures and interfaces}

The first step of the multiscale computational strategy consists of describing the structure as an assembly of simple components: substructures and interfaces [14] (see Figure 1). Each component has its own variables and equations. A substructure $\Omega_{\mathrm{E}}, E \in \mathbf{E}$, is subjected to the action of its environment (the neighboring interfaces) defined by a force distribution $\underline{\mathrm{F}}_{E}$ and a velocity distribution $\underline{\dot{W}}_{\mathrm{E}}$ on its boundary $\partial \Omega_{\mathrm{E}}$. An interface $\Gamma_{\mathrm{EE}^{\prime}}$ between two substructures $E$ et $E^{\prime}$ transfers both the velocity and the force distributions: $\underline{\mathrm{W}}_{\mathrm{E}}, \underline{\mathrm{W}}_{\mathrm{E}^{\prime}}$ and $\underline{\mathrm{F}}_{E}, \underline{\mathrm{F}}_{\mathrm{E}^{\prime}}$.

\subsection{Approximation - Choice of the spaces}

In practice, the interfaces and substructures are discretized in space classically using finite elements. The time interval $[0, T]$ being considered is decomposed into $\mathcal{T}_{h}=\left\{t_{0}=0, t_{1}, \ldots, t_{N}=T\right\}$. A space $\mathbf{S}^{[0, T]}$ with superscript $[0, T]$ designates the space of functions defined on $[0, T]$ which take values in $\mathbf{S}$. 

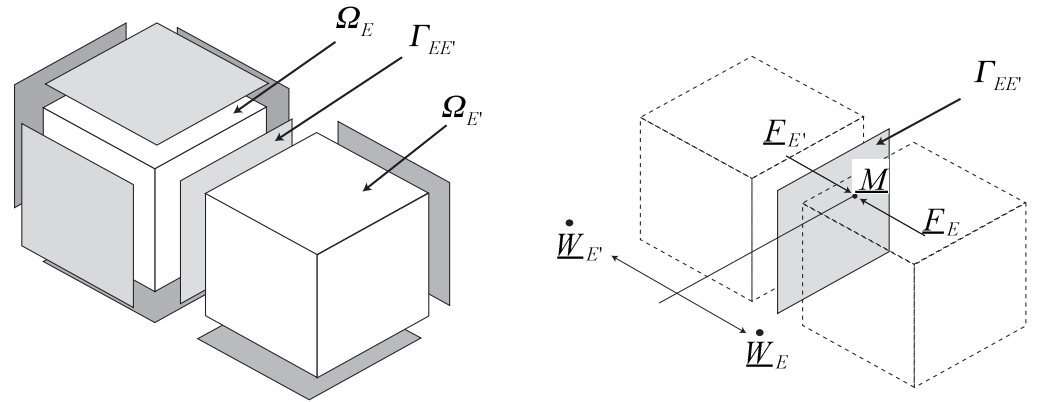

Fig. 1. Decomposition of a structure into substructures and interfaces

The spaces $\mathcal{W}_{h, \Gamma_{\mathrm{EE}}^{\prime}}^{[0, T]}$ and $\mathcal{F}_{h, \Gamma_{\mathrm{EE}}}^{[0, T]}$

The displacements $\underline{\mathrm{W}}$ and the forces $\underline{\mathrm{F}}$ at the interface $\Gamma_{\mathrm{EE}^{\prime}}$ belong to the

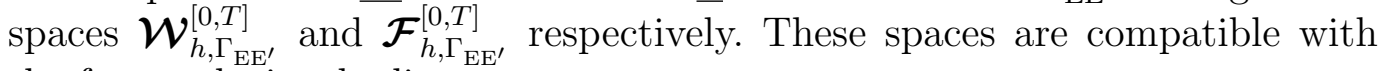
the force-velocity duality:

$$
\begin{aligned}
& (\underline{\mathrm{W}}, \underline{\mathrm{F}}) \quad \longmapsto \int_{\Gamma_{\mathrm{EE}}^{\prime} \times[0, T]} \underline{\mathrm{F}} \cdot \underline{\dot{\mathrm{W}}} d S d t \\
& \mathcal{W}_{h, \Gamma_{\mathrm{EE}^{\prime}}}^{[0, T]} \times \mathcal{F}_{h, \Gamma_{\mathrm{EE}^{\prime}}}^{[0, T]}
\end{aligned}
$$

and Proposition (1).

\section{Proposition 1}

$$
\begin{aligned}
& \left\{\underline{\mathrm{F}} \in \mathcal{F}_{h, \Gamma_{\mathrm{EE}^{\prime}}}^{[0, T]} ; \int_{\Gamma_{\mathrm{EE}^{\prime}} \times[0, T]} \underline{\mathrm{F}} \cdot \underline{\dot{\mathrm{W}}} d S d t=0, \forall \underline{\mathrm{W}} \in \mathcal{W}_{h, \Gamma_{\mathrm{EE}}^{\prime}}^{[0, T]}\right\} \Leftrightarrow \underline{\mathrm{F}}=0 \\
& \left\{\underline{\mathrm{W}} \in \mathcal{W}_{h, \Gamma_{\mathrm{EE}}^{\prime}}^{[0, T]} ; \int_{\Gamma_{\mathrm{EE}^{\prime}} \times[0, T]} \underline{\mathrm{F}} \cdot \underline{\underline{\mathrm{W}}} d S d t=0, \forall \underline{\mathrm{F}} \in \mathcal{F}_{h, \Gamma_{\mathrm{EE}}^{\prime}}^{[0, T]}\right\} \Leftrightarrow \underline{\mathrm{W}}=0
\end{aligned}
$$

Since the boundary of the substructure is discretized using finite elements, it is consistent to take for $\underline{\mathrm{W}}$ and $\underline{\mathrm{F}}$ regular functions, e.g. constant values within each element. On the time level, we consider discontinuous functions at times $t_{i}, i \in\{0, \ldots, N\}$. This choice turns out to be very efficient, but it requires the expression of work to be redefined.

Let us introduce $\mathbf{Z}_{r}^{[0, T]}$, the space of polynomial functions of degree $r$ on each $\left.\mathrm{I}_{i}=\right] t_{i}, t_{i+1}[, i \in\{0, \ldots, N-1\}$, with possible discontinuities. Then, for $(a, b) \in$ $\mathbf{Z}_{r}^{[0, T]}$, the expression of work becomes: 


$$
\int_{[0, T]} a \dot{b} d t=\sum_{i=0}^{N-1} \int_{\mathrm{I}_{i}} a \dot{b} d t+a\left(t_{i}^{+}\right)\left(b\left(t_{i}^{+}\right)-b\left(t_{i}^{-}\right)\right)
$$

where $b\left(t_{0}^{-}\right)=b_{0}$ is the initial condition .

This definition is associated with Galerkin discontinuous method [6]. A space $\mathbf{S}_{h}^{[0, T]}$ is said of type $\mathbf{Z}_{r}^{[0, T]}$ if

$$
\mathbf{S}_{h}^{[0, T]}=\left\{x:[0, T] \rightarrow \mathbf{X}_{h} / \forall i \in\{0, \ldots, N-1\}, x_{\mid I_{i}}=\sum_{k=0}^{r} x_{i, k} t^{k}, x_{i, k} \in \mathbf{S}_{h}\right\}
$$

If the spaces are of type $\mathbf{Z}_{r}^{[0, T]}$, the work is taken in the sense of (4). Let us observe that Proposition (1) is still verified.

Spaces $\mathbf{e}_{h, E}^{[0, T]}$ and $\mathbf{f}_{h, E}^{[0, T]}$

$\left(\boldsymbol{\sigma}_{E}, \mathbf{Y}_{E}\right)$ and $\left(\varepsilon_{p_{E}}, \mathbf{X}_{E}\right)$ belong to $\mathbf{f}_{h, E}^{[0, T]}$ and $\mathbf{e}_{h, E}^{[0, T]}$ respectively; these spaces are defined such that they are compatible with the dissipation bilinear form on $E$ :

$$
\int_{\Omega_{\mathrm{E}} \times[0, T]}\left\{\operatorname{Tr}\left[\boldsymbol{\sigma} \dot{\varepsilon}_{p}\right]-\mathbf{Y} \cdot \dot{\mathbf{X}}\right\} d \Omega d t
$$

On the space level, a classical finite element discretization is used. On the time level, spaces of type $\mathbf{Z}_{r}^{[0, T]}$ are used. The dissipation is taken in the sense of (4).

The following additional notations are introduced: $\mathbf{Y}_{E}$ and $\mathbf{X}_{E}$ belong to $\mathcal{Y}_{h, E}^{[0, T]}$ and $\mathcal{X}_{h, E}^{[0, T]}$ respectively; $\mathbf{s}_{E}=\left(\varepsilon_{p_{E}}, \mathbf{X}_{E}, \underline{\mathrm{W}}_{E}, \boldsymbol{\sigma}_{E}, \mathbf{Y}_{E}, \underline{\mathrm{F}}_{E}\right)$ indicates the set of variables associated with substructure E. The corresponding space is designated by $\mathcal{S}_{h, E}^{[0, T]}$.

\subsection{Reformulation of the reference problem}

First of all, it is necessary to clarify the sense in which the equilibrium equations are verified. Considering the space of the virtual displacements $\mathcal{U}_{h, E}^{[0, T]}$, let us write:

$$
\begin{aligned}
& \mathbf{F}_{h, E, a d}^{[0, T]}=\left\{\left(\boldsymbol{\sigma}_{E}, \underline{\mathrm{F}}_{E}\right) \in \mathbf{F}_{h, E}^{[0, T]} / \forall \underline{u}^{\star} \in \mathcal{U}_{E}^{[0, T]}, \int_{\Omega_{\mathrm{E}} \times[0, T]} \operatorname{Tr}\left[\boldsymbol{\sigma}_{E} \boldsymbol{\varepsilon}\left(\underline{\dot{u}}^{\star}\right)\right] d \Omega d t=\right. \\
& \left.\int_{\Omega_{\mathrm{E}} \times[0, T]} \underline{\mathrm{f}}_{d} \cdot \underline{\dot{u}}^{\star} d \Omega d t+\int_{\partial \Omega_{\mathrm{E}} \times[0, T]} \underline{\mathrm{F}}_{E} \cdot \underline{\dot{u}}_{\mid \partial \Omega_{\mathrm{E}}}^{\star} d S d t\right\}
\end{aligned}
$$


The space associated with $\underline{\mathrm{f}}_{d}=0$ is designated by $\mathbf{F}_{h, E, 0}^{[0, T]} \cdot\left(\boldsymbol{\sigma}_{E}, \underline{\mathrm{F}}_{E}\right)$ verify the equilibrium equations if and only if $\left(\boldsymbol{\sigma}_{E}, \underline{\mathrm{F}}_{E}\right) \in \mathbf{F}_{h, E, a d}^{[0, T]}$.

Now, let us write:

$$
\begin{gathered}
\mathbf{E}_{h, E, a d}^{[0, T]}=\left\{\left(\varepsilon_{E}, \underline{\mathrm{W}}_{E}\right) \in \mathbf{E}_{h, E}^{[0, T]} / \forall\left(\boldsymbol{\sigma}^{*}, \underline{\mathrm{F}}^{*}\right) \in \mathbf{F}_{h, E, 0}^{[0, T]},\right. \\
\left.\int_{\Omega_{\mathrm{E}} \times[0, T]} \operatorname{Tr}\left[\dot{\boldsymbol{\varepsilon}}_{E} \boldsymbol{\sigma}^{*}\right] d \Omega d t=\int_{\partial \Omega_{\mathrm{E}} \times[0, T]} \underline{\underline{\mathrm{W}}}_{\mathrm{E}} \cdot \underline{\mathrm{F}}^{*} d S d t\right\}
\end{gathered}
$$

$\left(\varepsilon_{E}, \underline{\mathrm{W}}_{E}\right)$ verify the compatibility equations if and only if $\left(\varepsilon_{E}, \underline{\mathrm{W}}_{E}\right) \in \mathbf{E}_{h, E, a d}^{[0, T]}$.

Definition $2 \mathbf{s}_{E}=\left(\varepsilon_{p_{E}}, \mathbf{X}_{E}, \underline{\mathrm{W}}_{E}, \boldsymbol{\sigma}_{E}, \mathbf{Y}_{E}, \underline{\mathrm{F}}_{E}\right) \in \mathcal{S}_{h, E}^{[0, T]}$ is E-admissible if it verifies:

- the equilibrium equations: $\left(\boldsymbol{\sigma}_{E}, \underline{\mathrm{F}}_{E}\right) \in \mathbf{F}_{h, E, a d}^{[0, T]}$

- the compatibility equations: $\left(\varepsilon_{E}, \underline{\mathrm{W}}_{E}\right) \in \mathbf{E}_{h, E, a d}^{[0, T]}$

- the state equations: $\boldsymbol{\varepsilon}_{E}-\boldsymbol{\varepsilon}_{p_{E}}=\mathbf{K}^{-1} \boldsymbol{\sigma}_{E}, \mathbf{Y}_{E}=\mathbf{\Lambda} \mathbf{X}_{E}$

- the initial conditions.

The corresponding space is designated by $\mathcal{S}_{h, E, a d}^{[0, T]}$.

The reference problem can be reformulated as follows:

Find $\mathbf{s}=\bigcup_{E \in \mathbf{E}} \mathbf{s}_{E}$ with $\mathbf{s}_{E}=\left(\varepsilon_{p_{E}}, \mathbf{X}_{E}, \underline{\mathrm{W}}_{E}, \boldsymbol{\sigma}_{E}, \mathbf{Y}_{E}, \underline{\mathrm{F}}_{E}\right) \in \mathcal{S}_{h, E}^{[0, T]}$ which verifies:

- the E-admissibility of $\mathbf{s}_{E}$

- the constitutive relation describing the state evolution:

$$
\int_{\Omega_{\mathrm{E}} \times[0, T]}\left(\left[\begin{array}{c}
\dot{\varepsilon}_{p E} \\
-\dot{\mathbf{X}}_{E}
\end{array}\right]-\mathbf{B}\left(\left[\begin{array}{c}
\boldsymbol{\sigma}_{E} \\
\mathbf{Y}_{E}
\end{array}\right]\right) \cdot\left[\begin{array}{c}
\boldsymbol{\sigma}^{*} \\
\mathbf{Y}^{*}
\end{array}\right]=0 \quad \forall\left(\boldsymbol{\sigma}^{*}, \mathbf{Y}^{*}\right) \in \mathbf{f}_{h, E}^{[0, T]}\right.
$$

- the behavior at the interfaces, of which the boundary conditions on $\partial_{1} \Omega$ and $\partial_{2} \Omega$ are particular cases.

\section{Description of quantities on the macroscale and on the microscale in the space-time domain $\Omega \times[0, T]$}

The distinction between the micro and macro levels is made only at the interfaces. For space, the macro scale is defined by the characteristic length of the interfaces, which is a priori much larger than the discretization scale. For 
time, the macro scale is associated with a coarse partitioning of the time interval $\mathcal{T}_{h^{\prime}}=\left\{t_{0}^{\prime}=0, t_{1}^{\prime}, \ldots, t_{N^{\prime}}^{\prime}=T\right\}$ being studied, which contains the previous partitioning $\mathcal{T}_{h}$. Its characteristic time $T_{M}=\sup _{i \in\left\{0, \ldots, N^{\prime}-1\right\}}\left(t_{i+1}^{\prime}-t_{i}^{\prime}\right)$ is much larger than the characteristic time $T_{m}=\sup _{i \in\{0, \ldots, N-1\}}\left(t_{i+1}-t_{i}\right)$.

Let us consider the interface $\Gamma_{\mathrm{EE}^{\prime}}$ between two substructures $\mathrm{E}$ and $\mathrm{E}$; we may freely choose the spaces in which the macro displacements and forces are sought, i.e.

$$
\mathcal{W}_{h, \Gamma_{\mathrm{EE}}^{\prime}}^{[0, T], M} \text { and } \mathcal{F}_{h, \Gamma_{\mathrm{EE}}}^{[0, T], M}
$$

These spaces are not necessarily subspaces of $\mathcal{W}_{h, \Gamma_{\mathrm{EE}^{\prime}}}^{[0, T]}$ and $\mathcal{F}_{h, \Gamma_{\mathrm{EE}}}^{[0, T]}$. Nevertheless, they are taken such that they are compatible with the force-velocity duality and Property (3).

\section{Proposition 3}

$$
\begin{aligned}
& \left\{\underline{\mathrm{F}}^{M} \in \mathcal{F}_{h, \Gamma_{\mathrm{EE}}^{\prime}}^{[0, T], M} ; \int_{\Gamma_{\mathrm{EE}^{\prime}} \times[0, T]} \underline{\mathrm{F}}^{M} \cdot \underline{\dot{\mathrm{W}}}^{M} d S d t=0, \forall \underline{\mathrm{W}}^{M} \in \mathcal{W}_{h, \Gamma_{\mathrm{EE}}^{\prime}}^{[0, T], M}\right\} \Leftrightarrow \underline{\mathrm{F}}^{M}=0 \\
& \left\{\underline{\mathrm{W}}^{M} \in \mathcal{W}_{h, \Gamma_{\mathrm{EE}}^{\prime}}^{[0, T], M} ; \int_{\Gamma_{\mathrm{EE}^{\prime}} \times[0, T]} \underline{\mathrm{F}}^{M} \cdot \underline{\dot{\mathrm{W}}}^{M} d S d t=0, \forall \underline{\mathrm{F}}^{M} \in \mathcal{F}_{h, \Gamma_{\mathrm{EE}}^{\prime}}^{[0, T], M}\right\} \Leftrightarrow \underline{\mathrm{W}}^{M}=0
\end{aligned}
$$

For example, one could take, on the space level, affine functions on $\Gamma_{\mathrm{EE}^{\prime}}$ for $\underline{\mathrm{W}}^{M}$ and $\underline{\mathrm{F}}^{M}$ and, on the time level, functions of type $\mathbf{Z}_{p}^{[0, T]}$ (polynomials of degree $\mathrm{p}$ in the macro intervals $\left.\mathrm{I}_{i}^{M}=\right] t_{i}^{\prime}, t_{i+1}^{\prime}\left[, i \in\left\{0, \ldots, N^{\prime}-1\right\}\right.$ ) for the grid $\mathcal{T}_{h^{\prime}}$. The only constraint is that the rigid body modes on $\partial \Omega_{\mathrm{E}}$ must be included in the space of the macro displacements. Finally, we write $\underline{\mathrm{W}}^{M}$ and $\underline{\mathrm{F}}^{M}$ on $\Gamma_{\mathrm{EE}^{\prime}} \times \mathrm{I}_{i}^{M}$ in the form $\sum_{k, l} \alpha_{k, l} \underline{e}_{k}^{M}(\underline{M}) f_{l}^{M}(t)$, for which a choice of basis functions $\underline{e}_{k}^{M}$ and $f_{l}^{M}$ is represented in Figures 2 and 3.

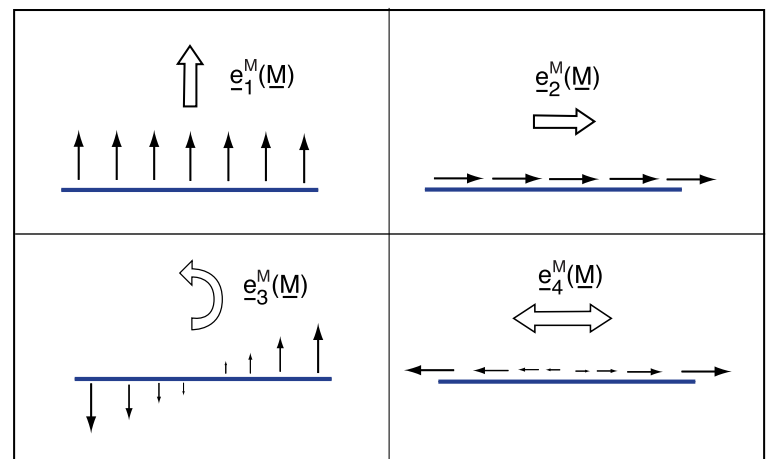

Fig. 2. Space level: affine basis functions $\left\{\underline{e}_{k}^{M}\right\}_{k \in\{1, \ldots, 4\}}$ for an interface $\Gamma_{\mathrm{EE}^{\prime}}$

The rationale adopted for the definition of the macro quantities is physically 


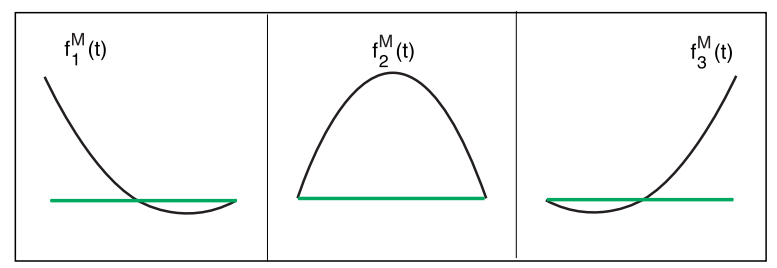

Fig. 3. Time level: quadratic basis functions $(p=2)\left\{f_{l}^{M}\right\}_{l \in\{1, \ldots, 3\}}$, in a macro interval $\mathrm{I}_{i}^{M}$

sound: these quantities are mean values in space as well as in time. More precisely, they are the best approximations in the sense of the work bilinear form (3). Due to Property (3), they are uniquely defined:

Definition 4 The macro parts $\left(\underline{\mathrm{W}}^{M}, \underline{\mathrm{F}}^{M}\right) \in \mathcal{W}_{h, \Gamma_{\mathrm{EE}}^{\prime}}^{[0, T], M} \times \mathcal{F}_{h, \Gamma_{\mathrm{EE}}^{\prime}}^{[0, T], M}$ of $(\underline{\mathrm{W}}, \underline{\mathrm{F}}) \in$

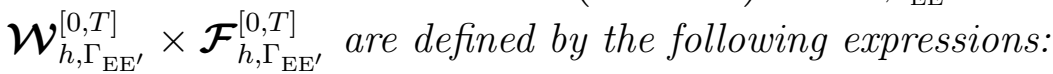

$$
\begin{aligned}
& \underline{\mathrm{W}}^{M} \in \mathcal{W}_{h, \Gamma_{\mathrm{EE}}^{\prime}}^{[0, T], M} ; \int_{\Gamma_{\mathrm{EE}}^{\prime} \times[0, T]}\left(\underline{\dot{\mathrm{W}}}^{M}-\underline{\dot{\mathrm{W}}}\right) \cdot \underline{\mathrm{F}}^{M^{*}} d S d t=0 ; \forall \underline{\mathrm{F}}^{M^{*}} \in \mathcal{F}_{h, \Gamma_{\mathrm{EE}}^{\prime}}^{[0, T], M} \\
& \underline{\mathrm{F}}^{M} \in \mathcal{F}_{h, \Gamma_{\mathrm{EE}}^{\prime}}^{[0, T], M} ; \int_{\Gamma_{\mathrm{EE}^{\prime}} \times[0, T]}\left(\underline{\mathrm{F}}^{M}-\underline{\mathrm{F}}\right) \cdot \underline{\mathrm{W}}^{M^{*}} d S d t=0 ; \forall \underline{\mathrm{W}}^{M^{*}} \in \mathcal{W}_{h, \Gamma_{\mathrm{EE}}^{\prime}}^{[0, T], M}
\end{aligned}
$$

Consequently, the micro parts are:

$$
\underline{\mathrm{F}}^{m}=\underline{\mathrm{F}}-\underline{\mathrm{F}}^{M} \text { and } \underline{\mathrm{W}}^{m}=\underline{\mathrm{W}}-\underline{\mathrm{W}}^{M}
$$

and the scales are uncoupled as follows:

$$
\int_{\Gamma_{\mathrm{EE}}^{\prime} \times[0, T]} \underline{\mathrm{F}} \cdot \underline{\dot{\mathrm{W}}} d S d t=\int_{\Gamma_{\mathrm{EE}} \times[0, T]}\left(\underline{\mathrm{F}}^{M} \cdot \underline{\dot{\mathrm{W}}}^{M}+\underline{\mathrm{F}}^{m} \cdot \underline{\dot{\mathrm{W}}}^{m}\right) d S d t
$$

This partitioning, extended to the set of interfaces, leads to the spaces $\mathcal{W}_{h}^{[0, T], M}$, $\mathcal{W}_{h}^{[0, T], m}, \mathcal{F}_{h}^{[0, T], M}$ and $\mathcal{F}_{h}^{[0, T], m}$.

Another important feature of the multiscale computational strategy presented here is that the transmission conditions at the interfaces are partially verified a priori. The macro forces are required to systematically verify the transmission conditions, including the boundary conditions. These conditions remain unchanged under unilateral contact. The corresponding space is designated by $\mathcal{F}_{h, a d}^{[0, T], M}$.

Remark 5 Relation (9) is independant of our homogenization procedure. We will see later that, taking the interfaces of a cell and integrating over them, 
the micro work can be written in terms of the data and of macro quantities.

\section{Multiscale computational strategy with space and time homog- enization}

\subsection{Principle}

Partial verification a priori of the transmission conditions at the interfaces leads to the following reformulation of the reference problem: Find $\mathbf{s}=\bigcup_{E \in \mathbf{E}} \mathbf{s}_{E}$ with $\mathbf{s}_{E} \in \mathcal{S}_{h, E}^{[0, T]}$ which verifies:

$$
\begin{aligned}
& \mathbf{A}_{d} \| \begin{array}{l}
\| \\
-\mathbf{s}_{E} \text { is E-admissible, } \mathrm{E} \in \mathbf{E} \\
-\bigcup_{E \in \mathbf{E}}\left\{\underline{\mathrm{F}}_{E}^{M}\right\} \in \mathcal{F}_{h, a d}^{[0, T], M}
\end{array} \\
& \mathbf{\Gamma} \| \text { the constitutive relation describing the state evolution (7) } \\
& - \text { the behavior at the interfaces }
\end{aligned}
$$

$\boldsymbol{\Gamma}$ represents a set of (possibly nonlinear) equations which are local in the space and time variables. $\mathbf{A}_{d}$ is a set of global linear equations. With this partitioning, it is possible to apply the LATIN method, a general computational strategy for time-dependent nonlinear problems which operates globally over the entire time-space domain. This method, described in [14], relies on some remarkable properties which are verified by most models encountered in structural mechanics. Figure 4 shows the scheme for one iteration, which consists of two steps.

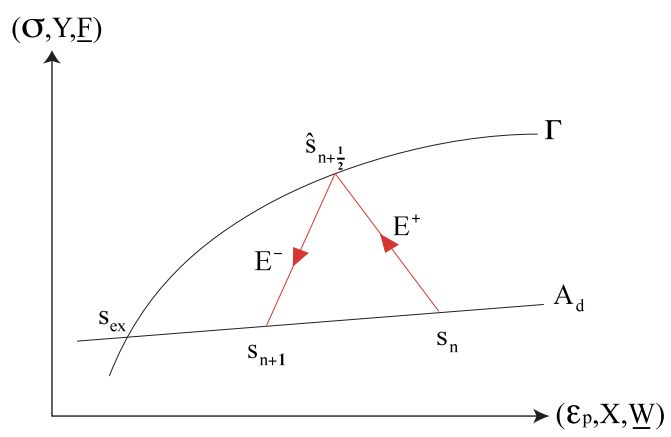

Fig. 4. The scheme for one iteration 


\subsection{The local stage at Iteration $n$}

$\mathbf{s}_{n}=\bigcup_{E \in \mathbf{E}} \mathbf{s}_{E_{n}}$ is given. The problem consists of building $\widehat{\mathbf{s}}_{n+1 / 2} \in \boldsymbol{\Gamma}$ knowing $\mathbf{s}_{n} \in \mathbf{A}_{d}$. $\left(\widehat{\mathbf{s}}_{n+\frac{1}{2}}-\mathbf{s}_{n}\right)$ must follow a search direction $\mathbf{E}^{+}$: more precisely, for each substructure $\mathrm{E}$ and each interface $\Gamma_{\mathrm{EE}^{\prime}}, \widehat{\mathbf{s}}_{n+1 / 2}$ must verify:

$$
\begin{aligned}
& \forall\left(\boldsymbol{\sigma}^{*}, \mathbf{Y}^{*}\right) \in \mathbf{f}_{h, E}^{[0, T]}, \\
& \int_{\Omega_{\mathrm{E}} \times[0, T]}\left\{\left[\begin{array}{c}
\dot{\widehat{\boldsymbol{\varepsilon}}}_{p E}-\dot{\boldsymbol{\varepsilon}}_{p E} \\
-\left(\dot{\widehat{\mathbf{X}}}_{E}-\dot{\mathbf{X}}_{E}\right)
\end{array}\right]+\mathbf{H}\left[\begin{array}{c}
\widehat{\boldsymbol{\sigma}}_{E}-\boldsymbol{\sigma}_{E} \\
\widehat{\mathbf{Y}}_{E}-\mathbf{Y}_{E}
\end{array}\right]\right\} \cdot\left[\begin{array}{c}
\boldsymbol{\sigma}^{*} \\
\mathbf{Y}^{*}
\end{array}\right] d \Omega d t=0 \\
& \forall \underline{\mathrm{F}}_{E}^{*} \in \mathcal{F}_{h, \Gamma_{\mathrm{EE}}}^{[0, T]}, \\
& \int_{\Gamma_{\mathrm{EE}^{\prime}} \times[0, T]}\left\{\mathbf{h}\left(\underline{\widehat{\mathrm{F}}}_{E}-\underline{\mathrm{F}}_{E}\right)-\left(\underline{\widehat{\mathrm{W}}}_{E}-\underline{\dot{\mathrm{W}}}_{E}\right)\right\} \cdot \underline{\mathrm{F}}_{E}^{*} d S d t=0
\end{aligned}
$$

where index $n$ has been dropped. $\mathbf{H}$ is a symmetric, positive definite operator which is a classical parameter of the method. $\mathbf{h}$ is another parameter of the method which can be interpreted as a micro flexibility of the interface. The local step presents no difficulty. The problems to be solved are local in the space and time variables and, therefore, lend themselves to the highest degree of parallelism.

\subsection{The linear stage at iteration $n$}

$\widehat{\mathbf{s}}_{n+1 / 2}=\bigcup_{E \in \mathbf{E}} \widehat{\mathbf{s}}_{E, n+1 / 2}$ is given. The problem consists of building $\mathbf{s}_{n+1} \in \mathbf{A}_{d}$ knowing $\widehat{\mathbf{s}}_{n+1 / 2} \in \boldsymbol{\Gamma}$. $\left(\mathbf{s}_{n+1}-\widehat{\mathbf{s}}_{n+1 / 2}\right)$ must follow a search direction $\mathbf{E}^{-}$: more precisely, for each substructure E:

$$
\begin{aligned}
& \forall\left(\boldsymbol{\sigma}^{*}, \mathbf{Y}^{*}\right) \in \mathbf{f}_{h, E}^{[0, T]}, \\
& \int_{\Omega_{\mathrm{E}} \times[0, T]}\left\{\left[\begin{array}{c}
\dot{\boldsymbol{\varepsilon}}_{p E}-\dot{\hat{\boldsymbol{\varepsilon}}}_{p E} \\
-\left(\dot{\mathbf{X}}_{E}-\dot{\widehat{\mathbf{X}}}_{E}\right)
\end{array}\right]-\mathbf{H}\left[\begin{array}{c}
\boldsymbol{\sigma}_{E}-\widehat{\boldsymbol{\sigma}}_{E} \\
\mathbf{Y}_{E}-\widehat{\mathbf{Y}}_{E}
\end{array}\right]\right\} \cdot\left[\begin{array}{c}
\boldsymbol{\sigma}^{*} \\
\mathbf{Y}^{*}
\end{array}\right] d \Omega d t=0 \\
& \forall \underline{\mathrm{F}}^{*} \in \mathcal{F}_{h}^{[0, T], m} \cup \mathcal{F}_{h, a d}^{[0, T], M}, \\
& \sum_{E \in \mathbf{E}} \int_{\partial \Omega_{\mathrm{E}} \times[0, T]}\left\{\mathbf{h}\left(\underline{\mathrm{F}}_{E}-\underline{\widehat{\mathrm{F}}}_{E}\right)+\left(\underline{\dot{\mathrm{W}}}_{E}-\underline{\widehat{\mathrm{W}}}_{E}\right)\right\} \cdot \underline{\mathrm{F}}_{E}^{*} d S d t=0
\end{aligned}
$$

where index $n$ has been dropped. One can easily prove that this problem is well-defined and has a unique solution if $\mathbf{h}$ and $\mathbf{H}$ are symmetric, positive definite operators. 
The problem can be split into two parts: a micro problem defined in each substructure and on each subinterval $\left[t_{i}, t_{i+1}\right], i \in\{0, \ldots, N-1\}$ and a global macro problem defined on the entire space-time domain $\Omega \times[0, T]$.

Most importantly, let us observe that Relation (13) can be rewritten as:

$$
\begin{aligned}
& \forall \underline{\mathrm{F}}^{*} \in \mathcal{F}_{h}^{[0, T]}, \sum_{E \in \mathbf{E}} \int_{\partial \Omega_{\mathrm{E}} \times[0, T]}\left\{\mathbf{h}\left(\underline{\mathrm{F}}_{E}-\underline{\widehat{\mathrm{F}}}_{E}\right)+\left(\underline{\dot{\mathrm{W}}}_{E}-\underline{\hat{\mathrm{W}}}_{E}\right)\right\} \cdot \underline{\mathrm{F}}_{E}^{*} d S d t= \\
& \sum_{E \in \mathbf{E}} \int_{\partial \Omega_{\mathrm{E}} \times[0, T]} \underline{\tilde{\mathrm{W}}}_{\mathrm{E}}^{M} \cdot \underline{\mathrm{F}}_{E}^{*} d S d t \\
& \forall \underline{\widetilde{\mathrm{W}}}^{M^{*}} \in \mathcal{W}_{h, 0}^{[0, T], M}, \\
& \sum_{E \in \mathbf{E}} \int_{\partial \Omega_{\mathrm{E}} \times[0, T]} \underline{\tilde{\mathrm{W}}}_{E}^{M^{*}} \cdot \underline{\mathrm{F}}_{E} d S d t=\sum_{E \in \mathbf{E}} \int_{\left(\partial \Omega_{\mathrm{E}} \cap \partial_{2} \Omega\right) \times[0, T]} \underline{\tilde{\mathrm{W}}}_{E}^{M^{*}} \cdot \underline{\mathrm{F}}_{d} d S d t
\end{aligned}
$$

where the Lagrange multiplier $\widetilde{\mathbb{W}}^{M}$ belongs to the space $\mathcal{W}_{h, a d}^{[0, T], M}$ of macro displacements which are continuous at the interfaces, are equal to zero on $\partial_{1} \Omega$ and are initially zero at $t_{0}{ }^{-}$. Let us note that, in this context, $\mathcal{W}_{h, 0}^{[0, T], M}=$ $\mathcal{W}_{h, a d}^{[0, T], M}$. Equation (15) expresses the admissibility of the macro forces.

4.3.1 The micro problem defined on substructure $E$ and its boundary $\partial \Omega_{\mathrm{E}}$ and on $[0, T]$

The micro problem associated with substructure E can be written as:

Find $\mathbf{s}_{E}=\left(\varepsilon_{p_{E}}, \mathbf{X}_{E}, \underline{\mathrm{W}}_{E}, \boldsymbol{\sigma}_{E}, \mathbf{Y}_{E}, \underline{\mathrm{F}}_{E}\right) \in \mathcal{S}_{h, E}^{[0, T]}$ which verifies:

- $\mathbf{s}_{E}$ is E-admissible

- the search direction (12) and (14).

This is a linear problem. Since the search direction (14) is local at each interface, the micro problem consists of a series of independent problems in each substructure. The kinematic admissibility equation and the state equations can be expressed in a weak sense as follows:

$$
\begin{aligned}
& \forall\left(\boldsymbol{\sigma}^{*}, \underline{\mathrm{F}}^{*}\right) \in \mathbf{F}_{h, E, 0}^{[0, T]}, \quad \forall \mathbf{Y}^{*} \in \mathcal{Y}_{h, E}^{[0, T]}, \\
& \int_{\Omega_{\mathrm{E}} \times[0, T]} \operatorname{Tr}\left[\left(\mathbf{K}^{-1} \dot{\boldsymbol{\sigma}}_{E}+\dot{\boldsymbol{\varepsilon}}_{p_{E}}\right) \boldsymbol{\sigma}^{*}\right] d \Omega d t+\int_{\Omega_{\mathrm{E}} \times[0, T]}\left(\boldsymbol{\Lambda}^{-1} \dot{\mathbf{Y}}_{E}-\dot{\mathbf{X}}_{E}\right) \cdot \mathbf{Y}^{*} d \Omega d t= \\
& \int_{\partial \Omega_{\mathrm{E}} \times[0, T]} \underline{\mathrm{W}}_{\mathrm{E}} \cdot \underline{\mathrm{F}}^{*} d S d t
\end{aligned}
$$

Introducing the search directions (12) and (14) into Equation (16), the micro problem becomes: 
Find $\left(\boldsymbol{\sigma}_{E}, \underline{\mathrm{F}}_{E}\right) \in \mathbf{F}_{h, E, a d}^{[0, T]}$ and $\mathbf{Y}_{E} \in \mathcal{Y}_{h, E}^{[0, T]}$ which verify the initial conditions and

$$
\begin{aligned}
& \forall\left(\boldsymbol{\sigma}^{*}, \underline{\mathrm{F}}^{*}\right) \in \mathbf{F}_{h, E, 0}^{[0, T]}, \quad \forall \mathbf{Y}^{*} \in \mathcal{Y}_{h, E}^{[0, T]}, \\
& \int_{\Omega_{\mathrm{E}} \times[0, T]} \operatorname{Tr}\left[\mathbf{K}^{-1} \dot{\boldsymbol{\sigma}}_{E} \boldsymbol{\sigma}^{*}\right] d \Omega d t+\int_{\Omega_{\mathrm{E}} \times[0, T]} \boldsymbol{\Lambda}^{-1} \dot{\mathbf{Y}}_{E} \cdot \mathbf{Y}^{*} d \Omega d t+ \\
& \int_{\Omega_{\mathrm{E}} \times[0, T]} \mathbf{H}\left[\begin{array}{c}
\boldsymbol{\sigma}_{E} \\
\mathbf{Y}_{E}
\end{array}\right] \cdot\left[\begin{array}{c}
\boldsymbol{\sigma}^{*} \\
\mathbf{Y}^{*}
\end{array}\right] d \Omega d t+\int_{\partial \Omega_{\mathrm{E}} \times[0, T]} \mathbf{h} \underline{\mathrm{F}}_{E} \cdot \underline{\mathrm{F}}^{*} d S d t= \\
& \int_{\partial \Omega_{\mathrm{E}} \times[0, T]}\left(\mathbf{h} \underline{\widehat{\mathrm{F}}}_{E}+\underline{\widehat{\widehat{\mathrm{W}}}}_{E}+\underline{\overrightarrow{\mathrm{W}}}_{\mathrm{E}}^{M}\right) \cdot \underline{\mathrm{F}}^{*} d S d t+ \\
& \int_{\Omega_{\mathrm{E}} \times[0, T]}\left(\mathbf{H}\left[\begin{array}{c}
\widehat{\boldsymbol{\sigma}}_{E} \\
\widehat{\mathbf{Y}}_{E}
\end{array}\right]-\left[\begin{array}{c}
\dot{\widehat{\boldsymbol{\varepsilon}}}_{p E} \\
-\dot{\widehat{\mathbf{X}}}_{E}
\end{array}\right]\right) \cdot\left[\begin{array}{c}
\boldsymbol{\sigma}^{*} \\
\mathbf{Y}^{*}
\end{array}\right] d \Omega d t
\end{aligned}
$$

This problem can be reformulated in terms of displacements and solved easily. The solution to the micro problem related to substructure E depends only on the known quantities $\underline{\mathrm{f}}_{d \mid \Omega_{\mathrm{E}}}, \widehat{\mathbf{s}}_{E}$, on the given initial condition and on the value of $\widetilde{\mathrm{W}}_{\mathrm{E}}^{M}$ at the boundary $\partial \Omega_{\mathrm{E}}$ which, at this stage, is unknown. One can prove that:

Proposition $\mathbf{6}$ If $\mathbf{H}$ and $\mathbf{h}$ are symmetric, positive definite operators, the micro problem defined in substructure $E$ and at its boundary has a unique solution such that:

$$
\begin{aligned}
\underline{\mathrm{F}}_{E \mid[0, T]}^{M} & =\mathbf{L}_{\mathrm{E}}^{F}\left(\underline{\widetilde{\mathrm{W}}}_{E \mid[0, T]}^{M}\right)+\widehat{\widehat{\mathrm{F}}}_{E, d \mid[0, T]}^{M} \\
\underline{\mathrm{W}}_{E \mid[0, T]}^{M} & =\mathbf{L}_{\mathrm{E}}^{W}\left(\underline{\widetilde{\mathrm{W}}}_{E \mid[0, T]}^{M}\right)+\underline{\widehat{\mathrm{W}}}_{E, d \mid[0, T]}^{M}
\end{aligned}
$$

where $\widetilde{\mathrm{W}}_{\mathrm{E}}^{M} \in \mathcal{W}_{h, E}^{[0, T], M}$. $\underline{\widehat{\mathrm{F}}}_{\mathrm{E}, d}$ and $\widehat{\mathrm{W}}_{\mathrm{E}, d}$ depend on $\underline{\mathrm{f}}_{d \mid \Omega_{\mathrm{E}}}, \widehat{\mathbf{s}}_{E}$ and on the initial conditions.

$\mathbf{L}_{\mathrm{E}}^{F}$ (respectively $\mathbf{L}_{\mathrm{E}}^{W}$ ) is a linear operator from $\mathcal{W}_{h, E}^{[0, T], M}$ to $\mathcal{F}_{h, E}^{[0, T], M}$ (respectively from $\mathcal{W}_{h, E}^{[0, T], M}$ to $\mathcal{W}_{h, E}^{[0, T], M}$ ) and can be interpreted as a homogenized behavior operator over the space-time substructure $\Omega_{\mathrm{E}} \times[0, T] . \mathbf{L}_{\mathrm{E}}^{F}$ and $\mathbf{L}_{\mathrm{E}}^{W}$ are calculated with zero initial conditions at a relatively low cost because $\widetilde{\mathrm{W}}_{\mathrm{E}}^{M}$ on $\partial \Omega_{\mathrm{E}} \times[0, T]$ depends on only a few scalar parameters. They represent the coupling effect among the different scales. A relation between the macro forces and the real macro displacements, which defines a more classical homogenized operator, can be easily deduced from relations (18) and (19). 
Let us recall that from the Lagrange multiplier $\widetilde{\mathrm{W}}_{\mathrm{E}}^{M}$, we can deduce all the quantities $\mathbf{s}_{E}$ on $\Omega_{\mathrm{E}} \times[0, T]$, i.e. there exists a localization operator $\mathbb{L}_{E}$, which is a linear operator from $\mathcal{W}_{h, E}^{[0, T], M}$ to $\mathcal{S}_{h, E}^{[0, T]}$ such that

$$
\mathbf{s}_{E}=\mathbb{L}_{E}\left(\underline{\widetilde{W}}_{E}^{M}\right)+\widehat{\mathbf{s}}_{E, d}^{M}
$$

where $\widehat{\mathbf{s}}_{E, d}^{M}$ depends on $\underline{f}_{d \mid \Omega_{\mathrm{E}}}, \widehat{\mathbf{s}}_{E}$ and on the initial conditions.

The micro problems defined on $\Omega_{\mathrm{E}} \times\left[t_{i}, t_{i+1}\right], \mathrm{E} \in \mathbf{E}$ and $i \in\{0, \ldots, N-$ $1\}$, are independent. Therefore, the local times can be different within the substructures. However, for each substructure E, the resolution of the micro problems must follow an incremental time scheme. This step lends itself to parallelism very well.

\subsubsection{The macro problem defined on $\Omega \times[0, T]$}

Relations (18) and (15) lead to the macro problem, which can be written as: Find $\underline{\widetilde{W}}^{M}=\bigcup_{E \in \mathbf{E}}\left\{\underline{\widetilde{W}}_{E}^{M}\right\} \in \mathcal{W}_{h, a d}^{[0, T], M}$ which verifies:

$$
\begin{aligned}
& \forall \underline{\widetilde{\mathrm{W}}}^{M^{*}} \in \mathcal{W}_{h, 0}^{[0, T], M}, \sum_{E \in \mathbf{E}} \int_{\partial \Omega_{\mathrm{E}} \times[0, T]} \underline{\overrightarrow{\mathrm{W}}}_{E}^{M^{*}} \cdot\left(\mathbf{L}_{\mathrm{E}}^{F}\left(\underline{\widetilde{\mathrm{W}}}_{E}^{M}\right)+\underline{\widehat{\mathrm{F}}}_{E, d}^{M}\right) d S d t= \\
& \sum_{E \in \mathbf{E}} \int_{\left(\partial \Omega_{\mathrm{E}} \cap \partial_{2} \Omega\right) \times[0, T]} \underline{\dot{\mathrm{W}}}_{E}^{M^{*}} \cdot \underline{\mathrm{F}}_{d} d S d t
\end{aligned}
$$

One can easily prove that the macro problem has a unique solution. Problem (21) can be formulated in terms of macro forces through a duality transformation.

The macro problem yields $\widetilde{\mathrm{W}}^{M}$. Then, using the micro problem again, or the localization operator $\mathbb{L}_{E}$ if it has been stored, one can determine $\mathbf{s}_{n+1}$ completely.

Macro quantities are defined only at the interfaces; by treating the medium as a Cosserat material, one can define macrostresses, macrostrains, ... inside a substructure E. Each cell is supposed to be homogeneous at the macroscale. So, from the generalized forces and displacements at the interfaces, macro quantities (displacements, directors) and conjugate quantities could be defined, which would lead to a non-conventional Cosserat-like material. This should be developped in a forecoming paper. 


\subsection{Reformulation of the linear stage at iteration $n$}

If the number of macro intervals increases, the cost of calculating the homogenized operator $\mathbf{L}_{\mathrm{E}}$ and solving the macro problem can become prohibitive. It is possible to reformulate the linear stage to circumvent this problem. Due to the use of Galerkin discontinuous method on the time level, the macro quantities (4) are defined independently in each macro interval $\mathrm{I}_{i}^{M}, i \in\left\{0, \ldots, N^{\prime}-1\right\}$ : the macro projection of displacements and forces on the interval $\mathrm{I}_{i}^{M}$ does not depend on the distribution of these displacements and forces in the other intervals $\mathrm{I}_{j}^{M}, j \neq i$.

Assuming that $\mathbf{s}$ and $\widetilde{\mathrm{W}}^{M}$ are known for $t<t_{i}^{\prime}$, let us define the following relation:

$$
\int_{\left[t_{i}^{\prime}, t_{i+1}^{\prime}\right]} a \dot{b} d t=\int_{\mathrm{I}_{i}^{M}} a \dot{b} d t+a\left(t_{i}^{\prime+}\right)\left(b\left(t_{i}^{\prime+}\right)-b\left(t_{i}^{\prime-}\right)\right)
$$

\subsubsection{The micro problem on $\Omega_{\mathrm{E}} \times\left[t_{i}^{\prime}, t_{i+1}^{\prime}\right]$}

The micro problem reduced to the interval $\left[t_{i}^{\prime}, t_{i+1}^{\prime}\right]$ can be written as: Find $\left(\boldsymbol{\sigma}_{E}, \underline{\mathrm{F}}_{E}\right) \in \mathbf{F}_{h, E, a d}^{\left[t_{i}^{\prime}, t_{i+1}^{\prime}\right]}$ and $\mathbf{Y}_{E} \in \mathcal{Y}_{h, E}^{\left[t_{t}^{\prime}, t_{i+1}^{\prime}\right]}$ which verify the initial conditions $\left(\boldsymbol{\sigma}_{E}\left(t_{i}^{\prime-}\right), \underline{\mathrm{F}}_{E}\left(t_{i}^{\prime-}\right)\right)$ and $\mathbf{Y}_{E}\left(t_{i}^{\prime-}\right)$ and Equation (17), in which the integrals over $[0, T]$ have been replaced by integrals over $\left[t_{i}^{\prime}, t_{i+1}^{\prime}\right]$.

The initial conditions $\left(\boldsymbol{\sigma}_{E}\left(t_{i}^{\prime-}\right), \mathbf{Y}_{E}\left(t_{i}^{\prime-}\right)\right)$ can be viewed as load sets on the "time interface" $\Omega_{\mathrm{E}} \times\left\{t_{i}^{\prime}\right\}$.

Proposition $\mathbf{7}$ If $\mathbf{H}$ and $\mathbf{h}$ are symmetric, positive definite operators, then $\forall E \in \mathbf{E}$ and $\forall i \in\left\{0, \ldots, N^{\prime}-1\right\}$ the micro problem defined on substructure $\Omega_{\mathrm{E}} \times\left[t_{i}^{\prime}, t_{i+1}^{\prime}\right]$ and its boundary $\partial \Omega_{\mathrm{E}} \times\left[t_{i}^{\prime}, t_{i+1}^{\prime}\right]$ has a unique solution such that:

$$
\underline{\mathrm{F}}_{E \mid \mathbf{I}_{i}^{M}}^{M}=\mathbf{L}_{E, i}^{F}\left(\underline{\mathrm{W}}_{E \mid \mathbf{I}_{i}^{M}}^{M}-\underline{\widetilde{W}}_{E}^{M}\left(t_{i}^{\prime-}\right)\right)+\underline{\underline{\mathrm{F}}}_{E, d \mid \mathbf{I}_{i}^{M}}^{M}
$$

where $\widetilde{\mathrm{W}}_{\mathrm{E}}^{M} \in \mathcal{W}_{h, E}^{\left[t_{i}^{\prime}, t_{i+1}^{\prime}\right], M} \cdot \underline{\widehat{\mathrm{F}}}_{E, d||_{i}^{M}}^{M}$ depends on $\underline{\mathrm{f}}_{d \mid \Omega_{\mathrm{E}} \times \mathrm{I}_{i}^{M}}, \widehat{\mathbf{s}}_{E}$ and on the initial conditions at $t_{i}^{\prime-}$.

Operator $\mathbf{L}_{E, i}^{F}$ is a homogenized behavior operator on the space-time substructure $\Omega_{\mathrm{E}} \times \mathrm{I}_{i}^{M}$ and can be calculated by solving a set of micro problems on $\Omega_{\mathrm{E}} \times \mathrm{I}_{i}^{M}$ with zero initial conditions at $t_{i}^{\prime-}$. These problems consist in taking successively, for $\left(\widetilde{\mathrm{W}}_{E \mid \mathrm{I}_{i}^{M}}^{M}-\underline{\widetilde{W}}_{E}^{M}\left(t_{i}^{\prime-}\right)\right)$, the macro basis functions on $\partial \Omega_{\mathrm{E}} \times \mathrm{I}_{i}^{M}$. The operator is defined in each substructure $\Omega_{\mathrm{E}} \times \mathrm{I}_{i}^{M}$ independently and its calculation can be parallelized in space as well as in time. Moreover, if the 
macro intervals $\mathbf{I}_{i}^{M}$ and the operators $\mathbf{H}$ and $\mathbf{h}$ on these intervals are identical, the calculation needs to be performed only once.

\subsubsection{The macro problem on $\Omega \times\left[t_{i}^{\prime}, t_{i+1}^{\prime}\right]$}

Find $\underline{\widetilde{W}}^{M}=\bigcup_{E \in \mathbf{E}}\left\{\widetilde{\widetilde{W}}_{E}^{M}\right\} \in \mathcal{W}_{h, a d}^{\left[t_{i}^{\prime}, t_{i+1}^{\prime}\right], M}$ which verifies the initial condition $\widetilde{\mathrm{W}}^{M}\left(t_{i}^{\prime-}\right)$ and

$$
\begin{aligned}
& \forall \underline{\widetilde{\mathrm{W}}}^{M^{*}} \in \mathcal{W}_{h, 0}^{\left[t_{i}^{\prime}, t_{i+1}^{\prime}\right], M}, \\
& \sum_{E \in \mathbf{E}} \int_{\partial \Omega_{\mathrm{E}} \times\left[t_{i}^{\prime}, t_{i+1}^{\prime}\right]}{\underline{\tilde{\mathrm{W}}_{E}}}_{E}^{M^{*}} \cdot\left(\mathbf{L}_{E, i}^{F}\left(\underline{\widetilde{W}}_{E}^{M}-\underline{\widetilde{\mathrm{W}}}_{E}^{M}\left(t_{i}^{\prime-}\right)\right)+\underline{\mathrm{F}}_{E, d}^{M}\right) d S d t= \\
& \sum_{E \in \mathbf{E}} \int_{\left(\partial \Omega_{\mathrm{E}} \cap \partial_{2} \Omega\right) \times\left[t_{i}^{\prime}, t_{i+1}^{\prime}\right]} \underline{\dot{\mathrm{W}}}_{E}^{M^{*}} \cdot \underline{\mathrm{F}}_{d} d S d t
\end{aligned}
$$

where the space $\mathcal{W}_{h, 0}^{\left[t_{i}^{\prime}, t_{i+1}^{\prime}\right], M}$ corresponds to $\mathcal{W}_{h, a d}^{\left[t_{i}^{\prime}, t_{i+1}^{\prime}\right], M}$ with zero value at $t_{i}^{\prime-}$. One can easily show that the macro problem has a unique solution.

\subsubsection{Adaptivity}

Due to causality, we must solve the linear stage incrementally over the macro time intervals. However, the reformulation of the problem enables a specific treatment on the time level. For example, one can choose, for the first few iterations, to solve the problem over the entire space-time domain; then, as the macro solution converges rapidly, one can calculate the following iterations on selected macro time intervals of interest.

Of course, this selection can be performed over a set of macro intervals, which leads to the definition of a homogenized operator over a small number of macro intervals.

\section{Convergence}

If the operator $\mathbf{B}$ which characterizes the material is monotonous and if the interfaces represent perfect connection, boundary conditions or unilateral contact without friction, the multiscale computational strategy verifies the usual assumptions of the LATIN method; indeed, we can use conjugate search directions and we have: 
Proposition $\mathbf{8}$ If $\mathbf{B}$ is monotonous and the interfaces are without friction, the following inequalities are verified:

$$
\begin{aligned}
& \bullet \forall\left(\widehat{\mathbf{s}}, \widehat{\mathbf{s}}^{\prime}\right) \in \boldsymbol{\Gamma}^{2}, \quad\left\langle\left(\widehat{\mathbf{s}}-\widehat{\mathbf{s}}^{\prime}\right),\left(\widehat{\mathbf{s}}-\widehat{\mathbf{s}}^{\prime}\right)\right\rangle \geqslant 0 \\
& \bullet \forall\left(\mathbf{s}, \mathbf{s}^{\prime}\right) \in \mathbf{A}_{d}^{2}, \quad\left\langle\left(\mathbf{s}-\mathbf{s}^{\prime}\right),\left(\mathbf{s}-\mathbf{s}^{\prime}\right)\right\rangle \leqslant 0
\end{aligned}
$$

where $\langle$,$\rangle is the bilinear form from \mathcal{S}_{h}^{[0, T]} \times \mathcal{S}_{h}^{[0, T]}$ to $\mathbb{R}$ such that:

$$
\begin{aligned}
& \left\langle\mathbf{s}, \mathbf{s}^{\prime}\right\rangle=\sum_{E \in \mathbf{E}}\left\{\int_{\Omega_{\mathrm{E}} \times[0, T]}\left(\operatorname{Tr}\left[\boldsymbol{\sigma}_{E} \dot{\boldsymbol{\varepsilon}}_{p E}^{\prime}\right]+\operatorname{Tr}\left[\boldsymbol{\sigma}_{E}^{\prime} \dot{\boldsymbol{\varepsilon}}_{p E}\right]-\mathbf{Y}_{E} \cdot \dot{\mathbf{X}}_{E}^{\prime}-\mathbf{Y}_{E}^{\prime} \cdot \dot{\mathbf{X}}_{E}\right) d \Omega d t\right. \\
& \left.-\int_{\partial \Omega_{\mathrm{E}} \times[0, T]}\left(\underline{\mathrm{F}}_{E} \cdot \underline{\underline{\mathrm{W}}}_{\mathrm{E}}^{\prime}+\underline{\mathrm{F}}_{E}^{\prime} \cdot \underline{\mathrm{W}}_{\mathrm{E}}\right) d S d t\right\}
\end{aligned}
$$

The above expression is taken in the sense of (4).

Following the proof of the monoscale strategy given in [14], we can prove that the iterative strategy converges. To ensure convergence for many types of material behavior, let us modify the linear stage: renaming $\widetilde{\mathbf{s}}_{n+1} \in \mathbf{A}_{d}$ the quantity previously denoted $\mathbf{s}_{n+1}$, we define $\mathbf{s}_{n+1}$ by the relation:

$$
\mathbf{s}_{n+1}=\mu \widetilde{\mathbf{s}}_{n+1}+(1-\mu) \mathbf{s}_{n}
$$

where $\mu$ is a relaxation parameter usually chosen equal to 0.8 .

Remark 9 This result does not work for softening material for which localization occurs. However, numerical experiments have been succesfully performed for the monoscale version.

\section{Approximation of the macro problem: introduction of a third scale}

If the number of macro space-time substructures $\Omega_{E} \times\left[t_{i}^{\prime}, t_{i+1}^{\prime}\right]$ is large, the cost of solving the macro problem can be prohibitive. For example, let us consider the response of a simple composite structure made of $100 \times 100$ cells and subjected to 100 loading cycles. With a classical macro space, the size of the macro problem is about $5 \times 10^{6}$, which is beyond our reasonable capabilities. Here, we propose to seek an approximate solution to the macro problem by introducing a third scale over the space-time domain. 


\subsection{The approximate macro problem}

Let us recall that the macro problem (21) yields the Lagrange multiplier $\widetilde{\mathrm{W}}^{M}$, from which we can deduce the complete solution of the linear stage. It converges towards zero along the iterations of the algorithm. In the case where the macro wavelength is rather large compared to the cell dimension, one can proceed to a further approximation for the macro quantities. Then, the idea is to seek an approximation to the solution of problem (21) in a "well-chosen" subspace $\mathcal{W}_{h, a d}^{[0, T], M, M}$ of $\mathcal{W}_{h, a d}^{[0, T], M}$. The approximate macro problem can be expressed as:

Find $\underline{\widetilde{W}}^{M, M}=\bigcup_{E \in \mathbf{E}}\left\{\underline{\widetilde{W}}_{E}^{M}\right\} \in \mathcal{W}_{h, a d}^{[0, T], M, M}$, which verifies:

$$
\begin{aligned}
& \forall \underline{\widetilde{\mathrm{W}}}^{M, M^{*}} \in \mathcal{W}_{h, 0}^{[0, T], M, M}, \sum_{E \in \mathbf{E}} \int_{\partial \Omega_{\mathrm{E}} \times[0, T]}{\underline{\dot{\mathrm{W}}_{E}^{M, M^{*}}}}_{E} \cdot\left(\mathbf{L}_{\mathrm{E}}\left(\widetilde{\widetilde{\mathrm{W}}}_{E}^{M, M}\right)+\underline{\widehat{\mathrm{F}}}_{E, d}^{M}\right) d S d t= \\
& \sum_{E \in \mathbf{E}} \int_{\left(\partial \Omega_{\mathrm{E}} \cap \partial_{2} \Omega\right) \times[0, T]} \underline{\overrightarrow{\mathrm{W}}}_{E}^{M, M^{*}} \cdot \underline{\mathrm{F}}_{d} d S d t
\end{aligned}
$$

The solution $\widetilde{\mathrm{W}}^{M, M}$ to Problem (26) is an approximation of the solution $\widetilde{\mathrm{W}}^{M}$ to Problem (21).

Remark 10 Another and more conventional version consists in approximating the macro problem, formulated in terms of macro forces.

\subsection{Description of the third scale}

\subsubsection{Three-level decomposition of $\Omega \times[0, T]$}

On the space level, the substructures $\Omega_{E}$ are gathered into separate macrocells $\Omega_{E^{M}}=\bigcup_{E \in \mathbf{E}^{M}} \Omega_{E}, E^{M} \in \mathbf{E}^{M}$ such that $\bigcup_{E^{M} \in \mathbf{E}^{M}} \Omega_{E^{M}}=\Omega$ (Figure 5).

On the space level, the third scale is defined by the characteristic length of the macrocells.

Similarly, one can easily introduce a third time scale.

\subsubsection{Description of the kinematics of the third scale}

The set of macrocells can be viewed as a coarse mesh of $\Omega$ made of linear quadrangles. An interface between two quadrangles is denoted $\Gamma_{p q}$, p and q being the interface's end nodes (Figure 6). The basis functions associated with node 


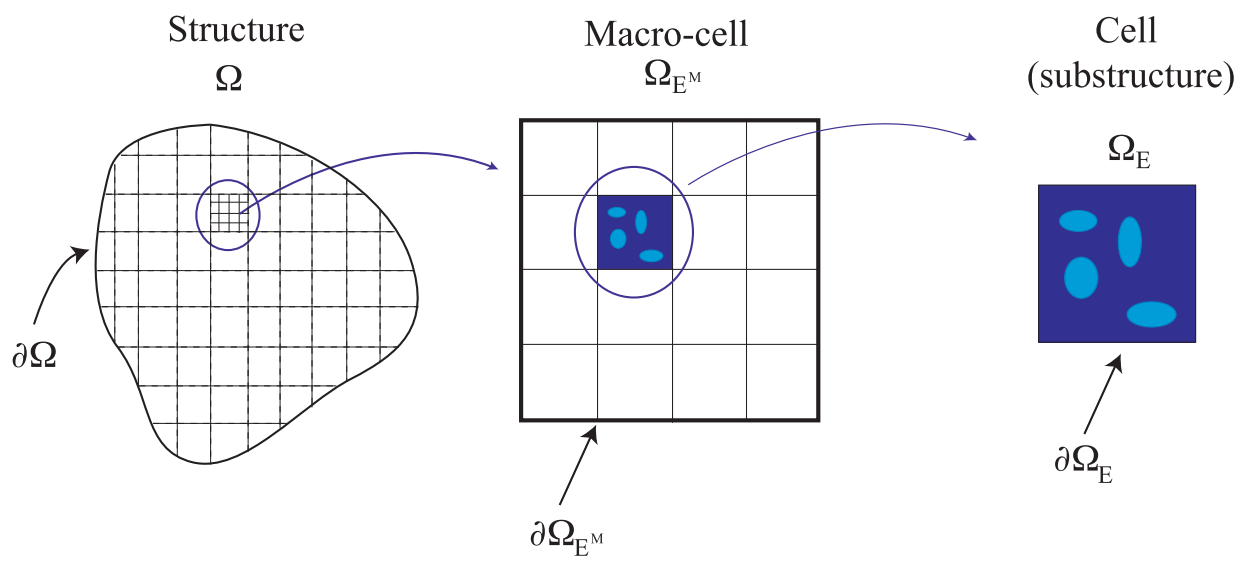

Fig. 5. Decomposition of the structure $\Omega$ into macrocells $\Omega_{E^{M}}$ and of the macrocells into substructures $\Omega_{E}$

p of this element are defined as usual and designated by $\left\{\underline{\varphi}_{p(1)}, \underline{\varphi}_{p(2)}\right\}$. We associate each side $\Gamma_{p q}$ of this quadrangle connecting nodes $\mathrm{p}$ and $\mathrm{q}$ with "supermacro" basis functions $\left\{\underline{e}_{p q, k}^{M, M}\right\}_{k \in\{1, \ldots, 6\}}$ defined on the interfaces of the macrocell $\Omega_{\mathrm{E}^{\mathrm{M}}}$ : the macro kinematics of the internal interfaces is interpolated from the global motions of the face $\Gamma_{p q}$ (including translations $\left\{\underline{\varphi}_{p(i)}+\underline{\varphi}_{q(i)}\right\}_{i \in 1,2}$, rotation $\left\{\underline{\varphi}_{q(2)}-\underline{\varphi}_{p(2)}\right\}$ and expansion $\left.\left\{\underline{\varphi}_{q(1)}-\underline{\varphi}_{p(1)}\right\}\right)$. This interpolation is weighted by the orientation of the interfaces. Figure 7 illustrates these supermacro basis functions.

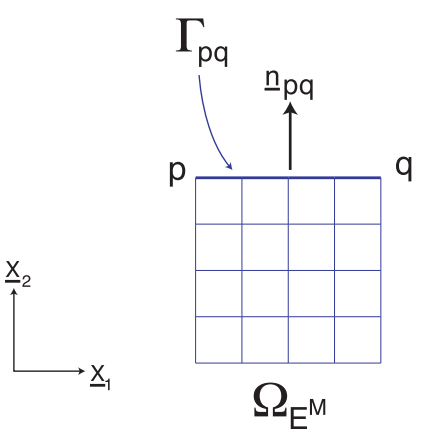

Fig. 6. The macrocell $\Omega_{\mathrm{E}^{\mathrm{M}}}$

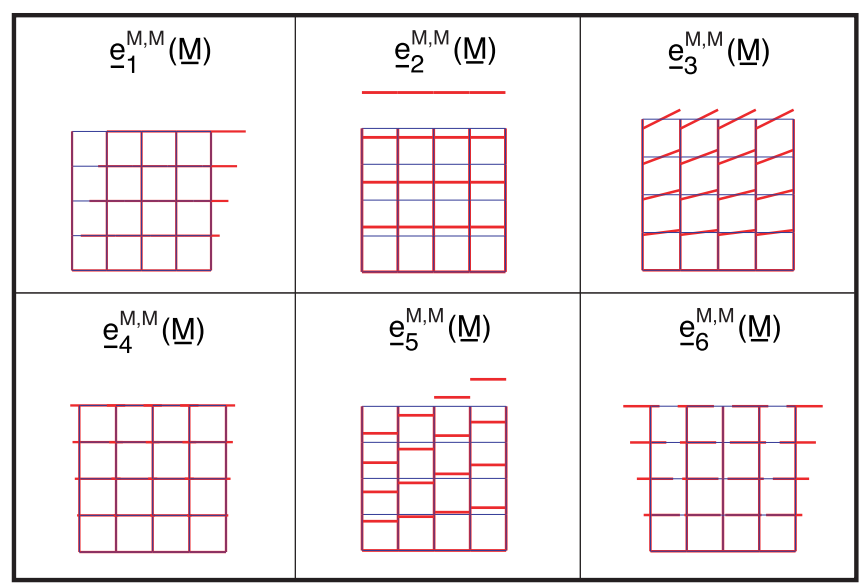

Fig. 7. "Super-macro" basis functions associated on the space level with the interface $\Gamma_{p q}$ of a macrocell $\Omega_{\mathrm{E}^{\mathrm{M}}}$ 


\section{Example 1: Three-scale modeling of a composite structure under tension and bending loads}

\subsection{Description of the problem}

Let us consider the 2D problem of a composite structure (Figure 8) fixed on the left and subjected to tension and bending $\underline{\mathrm{F}}_{d}$ loads on the right (Figure 9). We assume plane strain conditions. The in-plane dimensions are $30 \mathrm{~mm} \times 10 \mathrm{~mm}$ and the thickness is $1 \mathrm{~mm}$. The structure is made of two types of cells, denoted I and II. The materials are viscoelastic with Young's modulus $E_{i}$, Poisson's ratio $\nu_{i}$ and viscosity $\eta_{i}$. Their constitutive relations are such that $\mathbf{B}_{i}=\frac{1}{\eta_{i}} \mathbf{K}_{i}^{-1}$.

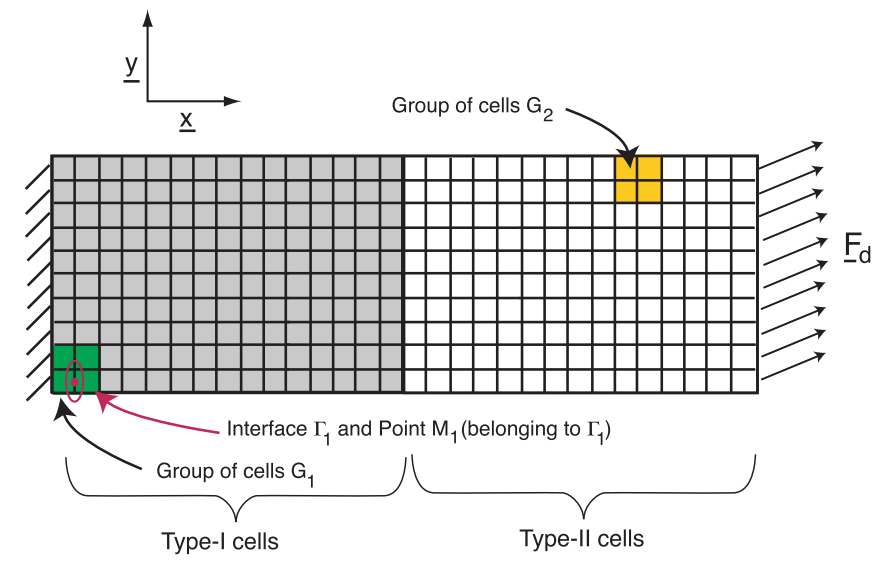

Fig. 8. Description of the problem: traction and bending loads on a composite structure with two types of cells

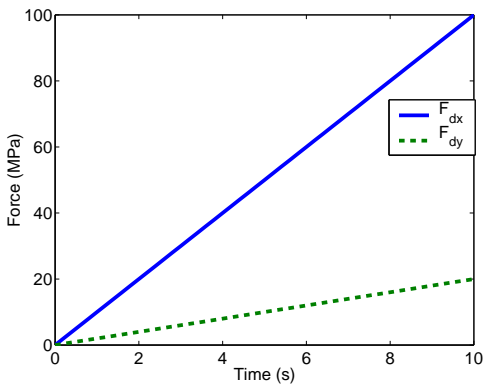

Fig. 9. Loading $\underline{F}_{d}$

Each cell is a substructure of the partitioned problem. The Type-II cell (Figure 11) is homogeneous with a Type- 1 material whose characteristics are $E_{1}=$ $1.3 * 10^{5} \mathrm{MPa}, \nu_{1}=0.45$ and $\eta_{1}=10 \mathrm{~s}$. It is meshed with $218 \mathrm{TRI} 3$. The Type-I cell (Figure 10) consists of a matrix of Type-1 material and inclusions of Type- 2 material whose characteristics are $E_{2}=1.3 * 10^{7} \mathrm{MPa}, \nu_{2}=0.2$ and $\eta_{2}=1000 \mathrm{~s}$. It is meshed with 1,068 TRI3. Each interface is meshed with 10 elements. The time interval being considered is $[0,10 s]$. On this example, we tested the three-scale approach in space: therefore, we discretized the time interval with only 10 micro subintervals $(N=10)$ and one macro interval $\left(N^{\prime}=1\right)$. The functions are of type $\mathbf{Z}_{0}^{[0, T]}$ on the micro grid $\tau_{h}$ and of type $\mathbf{Z}_{2}^{[0, T]}$ on the macro grid $\tau_{h^{\prime}}$. 


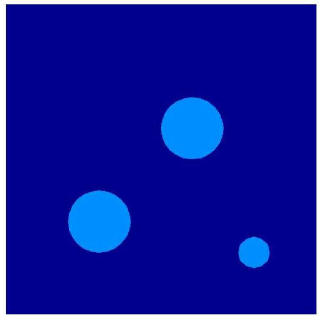

Fig. 10. Type-I cell: matrix of Type-1 material with inclusions of Type-2 material

\subsection{Presentation of the results}

Figures 12 and 13 show the spatial distribution of the micro and macro forces and displacements on the interface $\Gamma_{1}$ at $t=10 \mathrm{~s}$.

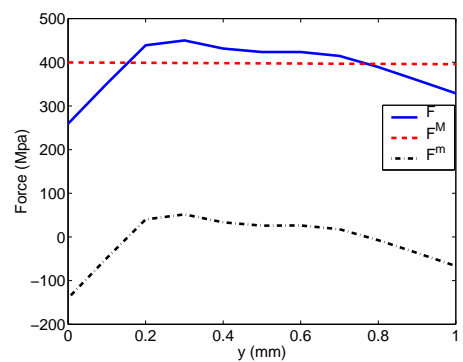

Fig. 12. Distribution of the projection on $\underline{x}$ of the micro and macro parts of force $\underline{\mathrm{F}}_{1}$ along the interface $\Gamma_{1}$ at time $t=10 \mathrm{~s}$

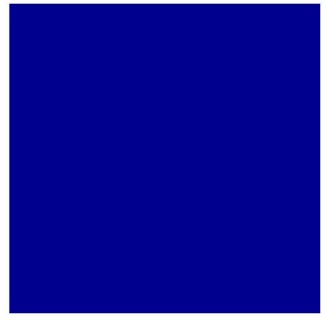

Fig. 11. Type-II cell: homogeneous, Type-1 material

Figures 14 and 15 show the time evolution of the micro and macro forces and displacements at point $M_{1}$. Let us observe that the macro forces and displacements represent the "mean" solution quite well, even in high-gradient zones.

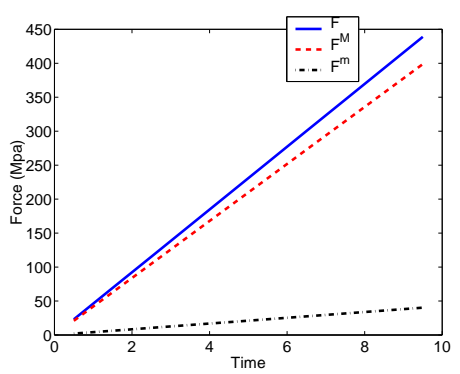

Fig. 14. Evolution of the projection on $\underline{x}$ of the micro and macro parts of force $\underline{\mathrm{F}}_{1}$ at point $M_{1}$

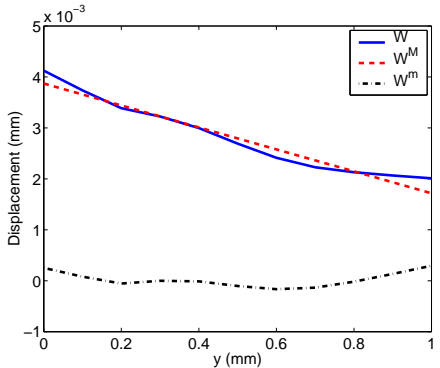

Fig. 13. Distribution of the projection on $\underline{x}$ of the micro and macro parts of displacement $\underline{\mathrm{W}}_{1}$ along $\Gamma_{1}$ at time $t=10 \mathrm{~s}$

Remark 11 For this academic example, it is clear that a classical monoscale approach could be used for the homogeneous part of the structure: it means 
that micro quantities can be taken as zero value.

Remark 12 Figure 17 shows the time evolution of the micro and macro forces and displacements at point $M_{1}$ in the case of a more complex traction loading with time perturbations (figure 16). For this example, the time interval being considered is $[0,30 s]$ and it is discretized with 150 micro subintervals $(N=$ $150)$ and three macro intervals $\left(N^{\prime}=3\right)$. The functions are of type $\mathbf{Z}_{0}^{[0, T]}$ on the micro grid $\tau_{h}$ and of type $\mathbf{Z}_{2}^{[0, T]}$ on the macro grid $\tau_{h^{\prime}}$.

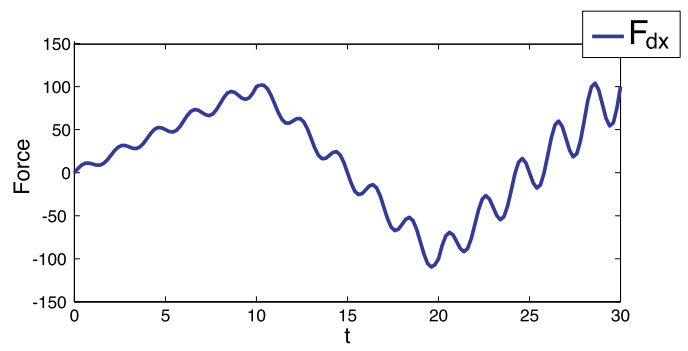

Fig. 16. Traction loading with time perturbations
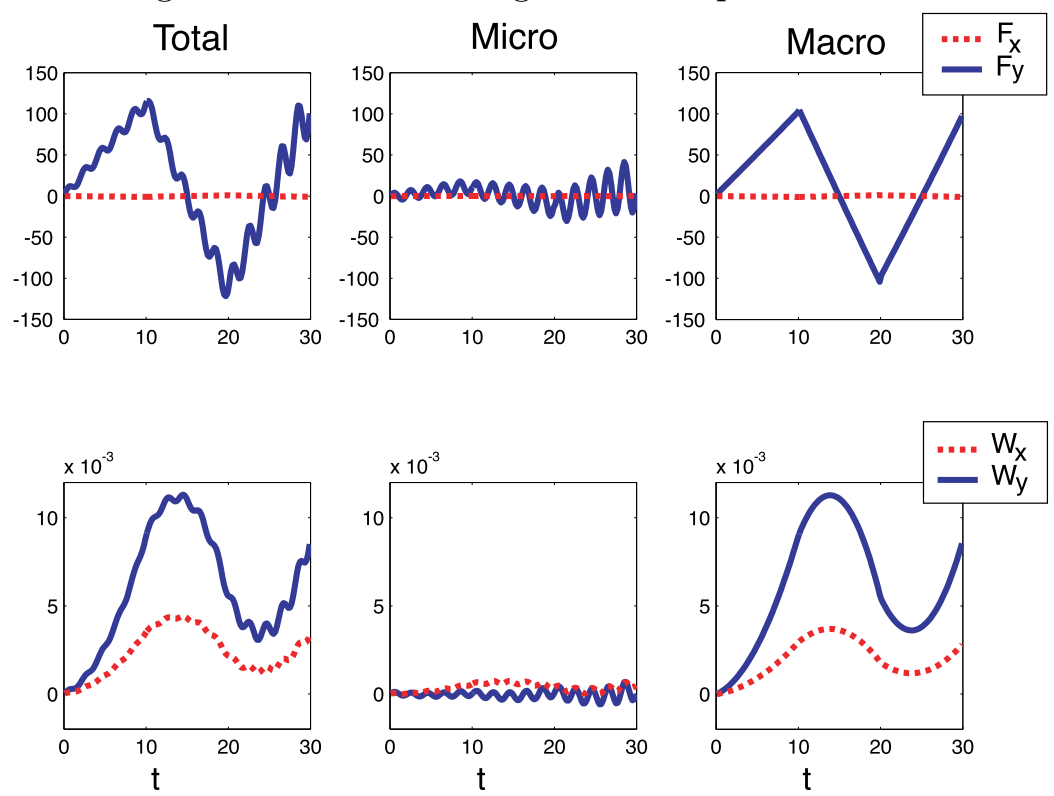

Fig. 17. Evolution of the projection on $\underline{x}$ and $y$ of the micro and macro parts of displacement ans force at point $M_{1}$

\subsection{Performance of the algorithm}

Figure 18 shows the evolution of the error with the number of iterations of the LATIN method. This error is based on the interface quantities $\underline{F}$ and $\underline{\mathrm{W}}$ and measures a distance between $\widehat{\mathbf{s}}_{n+1 / 2} \in \boldsymbol{\Gamma}$ and $\mathbf{s}_{n} \in \mathbf{A}_{d}$ at iteration $\mathrm{n}$. The algorithm converges towards a good solution very quickly (relative error less than 1/1,000 after 10 iterations). Figures 19 and 20 show the local 


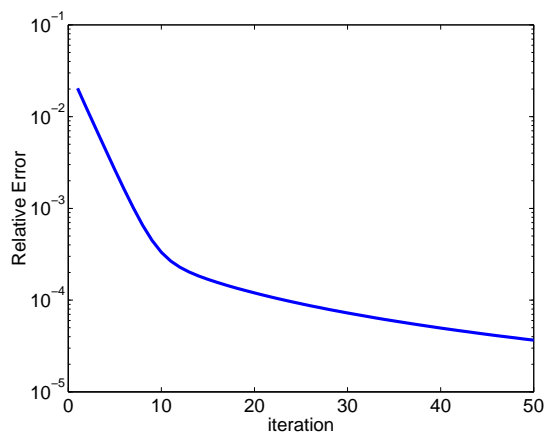

Fig. 18. Evolution with the number of iterations of an error based on interface quantities

displacements of the cell groups $G_{1}$ and $G_{2}$ and the macro displacements of the interfaces at time $t=10 \mathrm{~s}$ and at iteration 1 . In the low-gradient zone, the

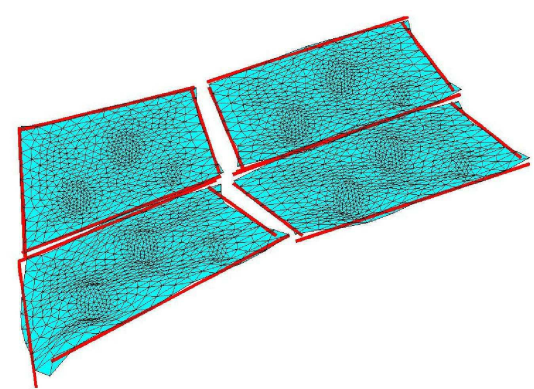

Fig. 19. Displacement and macro displacement of the interface $(\times 200)$ of cell group $G_{1}$ at time $t=10 \mathrm{~s}$ and at iteration 1

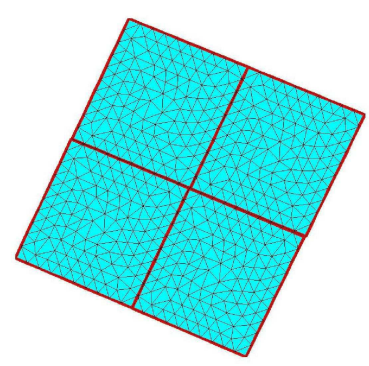

Fig. 20. Displacement and macro displacement of the interface $(\times 200)$ of cell group $G_{2}$ at time $t=10 \mathrm{~s}$ and at iteration 1

solution is already very good after the first iteration (which can be viewed as a simple macro calculation on the homogenized structure).

Let us note that the definition of cells is a choice. The total solution at convergence does not depend on this choice. However, the macro part could depend on it, particularly over high-gradient zones.

\subsection{The three-scale computational strategy}

Figures 21, 22, 23, 24 et 25 show the different super-macro meshes which were tested in this section. We considered only 2 time scales.

On Figure 26, we plotted the relative error with respect to a reference solution vs. the number of iterations for different super-macro meshes. The reference curve is the error obtained without the third scale, i.e. for a super-macro mesh identical to the macro mesh. Let us recall that the Lagrange multiplier $\widetilde{\mathrm{W}}^{M}$ 


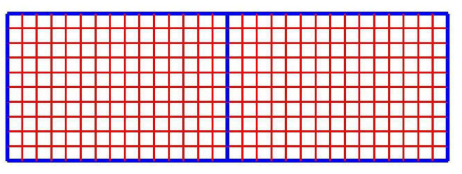

Fig. 21. Super-macro mesh $M M_{1}$

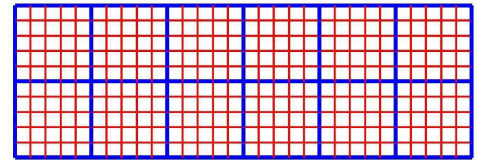

Fig. 23. Super-macro mesh $M M_{3}$

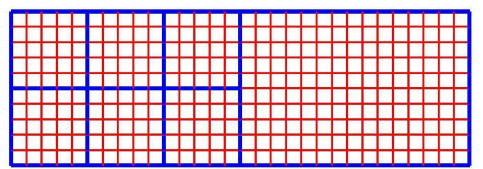

Fig. 22. Super-macro mesh $M M_{2}$

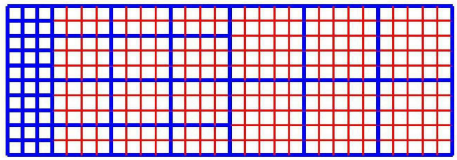

Fig. 24. Super-macro mesh $M M_{4}$

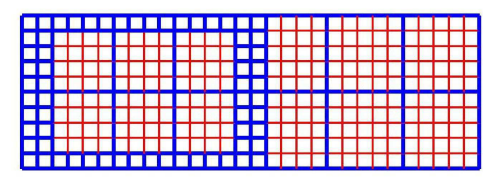

Fig. 25. Super-macro mesh $M M_{5}$

converges towards zero. Approximating it at each iteration does not change the solution at convergence but only the convergence rate of the algorithm. For example, taking for $\mathcal{W}_{h, a d}^{[0, T], M, M}$ the null space yields the classical monoscale strategy.

Table 1 shows the errors with the different meshes after 10 iterations and the corresponding sizes of the super-macro problems.

Table 1

Comparison of super-macro meshes: size of the macro problem and error after 10 iterations of the three-scale computational strategy

\begin{tabular}{|c|cc|}
\hline Super-macro mesh & Size of the macro problem & Error after 10 iterations \\
\hline$R e f$ & 7560 & $5.4 \times 10^{-2}$ \\
\hline$M M_{1}$ & 126 & $5.1 \times 10^{-1}$ \\
\hline$M M_{2}$ & 342 & $2.3 \times 10^{-1}$ \\
\hline$M M_{3}$ & 576 & $1.7 \times 10^{-1}$ \\
\hline$M M_{4}$ & 1548 & $5.8 \times 10^{-2}$ \\
\hline$M M_{5}$ & 2100 & $5.7 \times 10^{-2}$ \\
\hline
\end{tabular}

Mesh $M M_{1}$ is clearly inadequate. Meshes $M M_{2}$ and $M M_{3}$ performed quite similarly with an error of about $20 \%$ after 10 iterations. These similar performances show that the third scale worked very well in the low-gradient zones (the zones with Type-II cells). Mesh $M M_{4}$ differs simply in the refinement at the corner zones: this intuitive mesh gave very good results $(6 \%$ error after 10 iterations). Mesh $M M_{5}$, which is refined in the zone with the Type-I cells, per- 


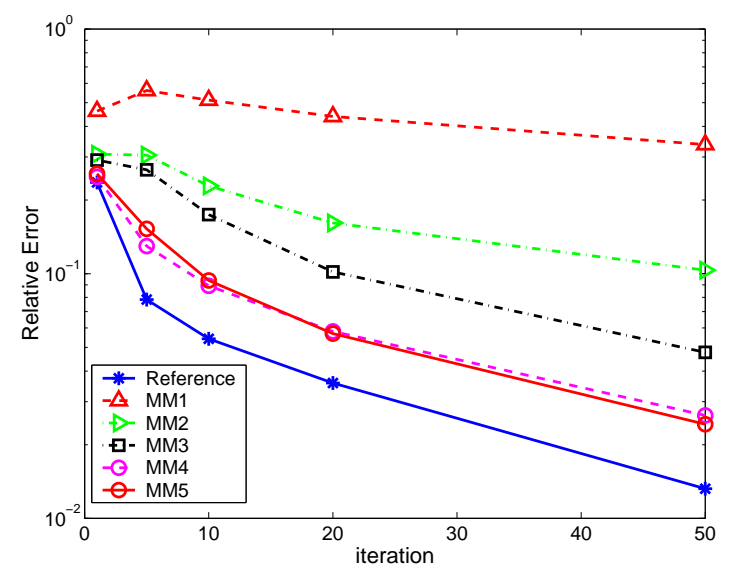

Fig. 26. Evolution of the error based on interface quantities vs. the number of iterations for different choices of super-macro meshes

formed much like the previous one. In conclusion, a "good" third-scale mesh can be coarse in the homogeneous low-gradient zones, a bit more refined in the heterogeneous zones and further refined in the high-gradient zones. With these guidelines, one obtains a good homogenized solution without modifying the convergence rate of the iterative method. In this case, with mesh $M M_{4}$, the size of the macro problem is divided by 5 . For problems with a much larger number of cells, the size of the macrocells would still be related to the macroscopic solution and, therefore, a macrocell could contain 100, 1,000 or more cells. Thus, one can hope to divide the size of the macro problem by $1,000,10,000$ or more.

Let us note that in a high-gradient zone (notch, ...), the third scale is not introduced.

Remark 13 We could have formulated the macro problem in terms of macro forces and approximated it. In this case, the solution at convergence depends on the choice of the third scale but the convergence rate should not depend on it. This version has not been tested yet.

\section{Approximation of micro problems: Radial loading approximation}

At each iteration and for each substructure, we need to solve micro problems which represent evolution equations defined on $\Omega_{\mathrm{E}} \times[0, T]$. The cost of solving these problems with standard incremental methods can be prohibitive, especially if the search direction associated with the linearized behavior $\mathbf{H}(t)$ is time-dependent. In this section, we introduce an approximation technique for the solution of these problems. This technique, commonly used in the LATIN method [14] (Point P3), defines an approximation based on generalized radial 
functions.

\subsection{Rewriting of the linear stage}

The linear stage at iteration $\mathrm{n}$ is rewritten in terms of the increment $\Delta \mathbf{s}=$ $\mathbf{s}_{n+1}-\mathbf{s}_{n}$. The initialization must be accurate with respect to the admissibility of the macro forces and the equilibrium of the substructures. A simple and sufficient way to fulfill this condition is to perform a linear elastic calculation for $\mathbf{s}_{0}$. The micro problem on substructure E becomes : Find $\Delta \mathbf{s}_{E} \in \mathcal{S}_{h, E, 0}^{[0, T]}$ which verifies the following relations, coming from the search direction:

$$
\begin{aligned}
& \forall\left(\boldsymbol{\sigma}^{*}, \mathbf{Y}^{*}\right) \in \mathbf{f}_{h, E}^{[0, T]}, \\
& \int_{\Omega_{\mathrm{E}} \times[0, T]}\left\{\left[\begin{array}{c}
\Delta \dot{\varepsilon}_{p E} \\
-\left(\Delta \dot{\mathbf{X}}_{E}\right)
\end{array}\right]-\mathbf{H}\left[\begin{array}{c}
\Delta \boldsymbol{\sigma}_{E} \\
\Delta \mathbf{Y}_{E}
\end{array}\right]-\hat{\boldsymbol{\Delta}}\right\} \cdot\left[\begin{array}{c}
\boldsymbol{\sigma}^{*} \\
\mathbf{Y}^{*}
\end{array}\right] d \Omega d t=0 \\
& \forall \underline{\mathrm{F}}^{*} \in \mathcal{F}_{h}^{[0, T]}, \int_{\partial \Omega_{\mathrm{E}} \times[0, T]}\left\{\mathbf{h}\left(\Delta \underline{\mathrm{F}}_{E}\right)+\left(\Delta \underline{\mathrm{W}}_{E}\right)-\underline{\hat{\delta}}\right\} \cdot \underline{\mathrm{F}}_{E}^{*} d S d t=0
\end{aligned}
$$

where $\hat{\boldsymbol{\Delta}}$ and $\underline{\hat{\delta}}$ are data and where $\mathcal{S}_{h, E, 0}^{[0, T]}$ corresponds to the space $\mathcal{S}_{h, E, a d}^{[0, T]}$ with homogeneous conditions.

\subsection{Concept of radial loading approximation}

Point P3 of the LATIN method consists in seeking an approximate solution to the previous micro problem as a sum of radial functions which are products of scalar functions of the time variable by functions of the space variable : for $E \in \mathbf{E}$,

$$
\begin{aligned}
& \left(\Delta \boldsymbol{\sigma}_{E}, \Delta \underline{\mathrm{F}}_{E}\right)(\underline{M}, t)=\sum_{k=1}^{m} c^{k}(t)\left(\mathbf{C}^{k}, \underline{\mathrm{G}}^{k}\right)(\underline{M}), \quad \Delta \mathbf{Y}_{E}(\underline{M}, t)=\sum_{k=1}^{m^{\prime}} d^{k}(t) \mathbf{D}^{k}(\underline{M}) \\
& \left(\Delta \boldsymbol{\varepsilon}_{E}, \Delta \underline{\mathrm{W}}_{E}\right)(\underline{M}, t)=\sum_{k=1}^{m^{\prime \prime}} e^{k}(t)\left(\mathbf{E}^{k}, \underline{\mathrm{Z}}^{k}\right)(\underline{M})
\end{aligned}
$$

Radial functions are choosen in the admissibible space $\mathcal{S}_{h, E, 0}^{[0, T]}: c^{k}, d^{k}$ and $e^{k}$ are functions of time of type $\mathbf{Z}_{r}^{[0, T]}$ with zero initial conditions, $\left(\mathbf{C}^{k}, \underline{\mathrm{G}}^{k}\right) \in \mathbf{F}_{h, E, 0}$, $\left(\mathbf{E}^{k}, \underline{\mathrm{Z}}^{k}\right) \in \mathbf{E}_{h, E, 0}$ and $\mathbf{D}^{k} \in \mathcal{Y}_{h, E}$. The state equations yield the following relations: 


$$
\begin{aligned}
& \Delta \boldsymbol{\varepsilon}_{p E}=\sum_{k=1}^{m^{\prime \prime}} e^{k}(t)\left(\mathbf{E}^{k}, \underline{\mathrm{Z}}^{k}\right)(\underline{M})-\sum_{k=1}^{m} c^{k}(t) \mathbf{K}^{-1}\left(\mathbf{C}^{k}, \underline{\mathrm{G}}^{k}\right)(\underline{M}) \\
& \Delta \mathbf{X}=\sum_{k=1}^{m^{\prime}} d^{k}(t) \boldsymbol{\Lambda}^{-1} \mathbf{D}^{k}(\underline{M})
\end{aligned}
$$

\subsection{Seeking the "best" approximation}

\subsubsection{Definition of the "best" approximation}

Relations (27) and (28) can be interpreted as linear constitutive relations. Due to the properties of operators $\mathbf{H}$ and $\mathbf{h}$, the micro problem can be reformulated as a minimization problem in the admissible space $\mathcal{S}_{h, E, 0}^{[0, T]}$ of the constitutive relation error $\mathbf{e}_{C R}\left(\Delta \mathbf{s}_{E}\right)$ defined by

$$
\begin{aligned}
\mathbf{e}_{C R}^{2}\left(\Delta \mathbf{s}_{E}\right)= & \left\|\left[\begin{array}{c}
\Delta \dot{\varepsilon}_{p E} \\
-\Delta \dot{\mathbf{X}}_{E}
\end{array}\right]-\mathbf{H}\left[\begin{array}{c}
\Delta \boldsymbol{\sigma}_{E} \\
\Delta \mathbf{Y}_{E}
\end{array}\right]-\hat{\Delta}\right\|_{\Omega_{\mathrm{E}} \times[0, T]}^{2}+ \\
& \left\|\mathbf{h} \Delta \underline{\mathrm{F}}_{E}+\Delta \underline{\dot{W}}_{E}-\underline{\hat{\delta}}\right\|_{\partial \Omega_{\mathrm{E}} \times[0, T]}^{2}
\end{aligned}
$$

where

$$
\begin{aligned}
\|\hat{\boldsymbol{\Delta}}\|_{\Omega_{\mathrm{E}} \times[0, T]}^{2} & =\int_{\Omega_{\mathrm{E}} \times[0, T]}\left(1-\frac{t}{T}\right) \hat{\boldsymbol{\Delta}} \cdot \mathbf{H}^{-1} \hat{\boldsymbol{\Delta}} d \Omega d t \\
\text { and } \quad\|\underline{\hat{\delta}}\|_{\Omega_{\mathrm{E}} \times[0, T]}^{2} & =\int_{\partial \Omega_{\mathrm{E}} \times[0, T]}\left(1-\frac{t}{T}\right) \underline{\hat{\delta}} \cdot \mathbf{h}^{-1} \underline{\hat{\delta}} d S d t
\end{aligned}
$$

The best radial functions are simply defined such that they minimize the constitutive relation error $\mathbf{e}_{C R}$. Formulated in this way, the approximation "makes sense". Details will be given in a following paper.

Let us note that the approximation of the solution to the linear stage still belongs to $\mathbf{A}_{d}$. Only the search direction of the algorithm is approximated.

\subsubsection{The resolution technique}

To solve the minization problem on the space of radial functions, we use an iterative scheme, proposed in [14], which consists in minimizing alternatively on time functions and space functions. Minimizing on time functions leads to a simple differential system of equations while minimizing on space functions leads to a "spatial" problem. In practice, only one function for each variable is 
calculated at the same time, i.e. $m=m^{\prime}=m^{\prime \prime}=1$, and the iterative scheme is stopped after two iterations.

Remark 14 Localization operator $\mathbb{L}_{E}$ and homogenized operators $\mathbf{L}_{\mathrm{E}}^{F}$ and $\mathbf{L}_{\mathrm{E}}^{W}$ can also be calculated with this approximation technique by taking $\hat{\boldsymbol{\Delta}}=0$ and successively, for $\underline{\hat{\delta}}$, the macro basis functions.

- Cost of the approximation

The advantage of such an approach is that because the spatial problem and the differential equation are uncoupled the cost of solving the linear stage is almost independent of the time integration scheme. Therefore, for the same cost, one can use robust implicit schemes on fine time grids, which would be unreasonably expensive with standard incremental methods.

Remark 15 Minimizing simultaneously on $(\mathbf{C}, \underline{\mathrm{G}}, \mathbf{D}, \mathbf{E}, \underline{\mathrm{Z}})$ leads to a "spatial" problem whose size is two times bigger than for the problem on an increment of a classical incremental method. An alternative iterative scheme consists in decoupling the minimization on $(\mathbf{C}, \underline{G}, \mathbf{D})$ from the minimization on $(\mathbf{E}, \underline{\mathrm{Z}})$.

- Reuse of functions

The functions of the space variable built using this approximation technique can be stored and systematically reused in the subsequent iterations of the LATIN method. The resolution of a micro problem begins with an initialization step which consists in solving a simple system of differential equations whose size is the number of spatial functions stored. This initialization step may yield good solutions to the micro problems directly. Thus, solving the micro problems becomes less and less costly as the iterations of the LATIN method proceed. Moreover, in the case of composite structures, the basis built as mentioned here is common to all identical substructures. Thus, one can expect that only a few spatial functions need to be calculated to solve the problem completely.

\section{Example 2: Solution of micro problems with the radial loading approximation}

\subsection{Description of the problem}

Let us consider the 2D problem of a composite structure (Figure 27) fixed on the left and subjected to tension and bending loads $\underline{\mathrm{F}}_{d}$ on the right (Figure 28). The in-plane dimensions are $25 \mathrm{~mm} \times 10 \mathrm{~mm}$ and the thickness is $1 \mathrm{~mm}$. We assume plane strain conditions. The structure is made of 40 identical cells. 
The cells consists of a matrix of Type- 1 material and inclusions of Type- 2 material. The materials are defined in example 1.

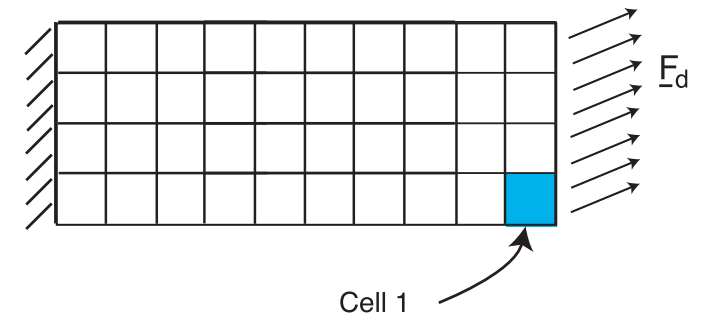

Fig. 27. Description of the problem: traction and bending loads on a composite structure composed of 48 cells

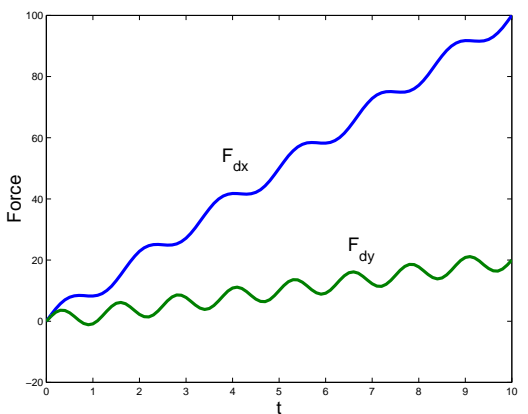

Fig. 28. Loading $\underline{\mathrm{F}}_{d}$

Each cell (Figure 29), which is a substructure of the partitioned problem, was meshed with 444 TRI3. Each interface was meshed with 8 elements.

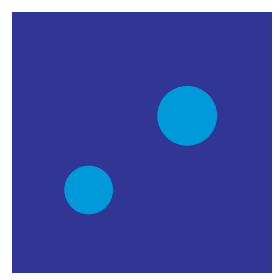

Fig. 29. Cell description: matrix of Type-1 material with inclusions of Type-2 material

The time interval $[0,10 s]$ being considered was discretized with 100 micro subintervals $(N=100)$ and one macro interval $\left(N^{\prime}=1\right)$. The functions are of type $\mathbf{Z}_{0}^{[0, T]}$ on the micro grid $\tau_{h}$ and of type $\mathbf{Z}_{2}^{[0, T]}$ on the macro grid $\tau_{h^{\prime}}$.

\subsection{Resolution of the micro problems}

To solve the micro problems, we used the radial loading approximation. At each iteration and for each substructure, we performed an initialization step using the space of the spatial functions previously stored. If this initialization was not sufficient (as shown by a test on the constitutive relation error), we solved the minimization problem of the constitutive relation error with the iterative scheme proposed previously, looking for one additional radial function for each variable, and performing only two iterations.

\subsection{Convergence of the algorithm}

Figure 30 shows the evolution of the error with respect to the number of iterations of the LATIN method. This error is based on the interface quantities 
$\underline{F}$ and $\underline{\mathrm{W}}$. We plotted convergence curves of the reference method, for which micro problems are solved exactly with a classical incremental scheme, and of the radial approximation, for which only one additional radial function per variable is calculated for each micro problem and stored in order to initialize the following ones. Let us note that the use of the approximation technique does not modify the global convergence of the algorithm and that, in practice, very few functions are needed to get an approximate solution.

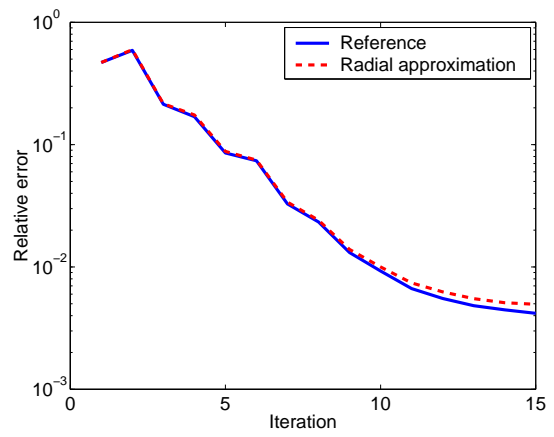

Fig. 30. Evolution of the error based on interface quantities with the number of iterations: comparison between the classical algortihm and the algorithm with the radial loading approximation

More details will be given in a forecoming paper.

\subsection{Illustration of the approximation}

Let us look at the solution for Cell 1. Figure 31 shows the corresponding stress field at time $t=10 \mathrm{~s}$.
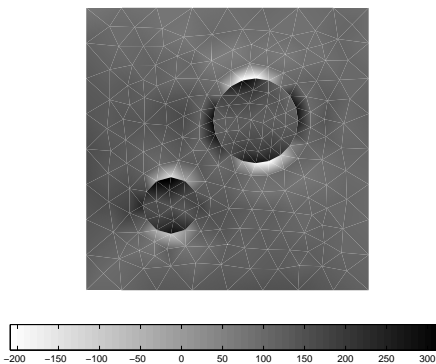

Fig. 31. $\boldsymbol{\sigma}$ in Cell 1 at time $t=10 \mathrm{~s}$

To illustrate the radial loading approximation, we solved the micro problem on cell 1 with data corresponding to the solution, i.e. $\hat{\Delta}=\dot{\boldsymbol{\varepsilon}}_{p E}-\mathbf{H} \boldsymbol{\sigma}_{E}$ and $\underline{\hat{\delta}}=\mathbf{h}_{E}+\underline{\mathrm{W}}_{E}$. Then, the exact solution of the micro problem is the solution itself. Figure 32 shows the stress field at time $t=10 \mathrm{~s}$ associated with the first three radial functions $\left\{c^{k}(10) \mathbf{C}^{k}\right\}_{k=1}^{3}$. Functions of the space variable are normalized. 
$c^{1}(10) C_{11}^{1}$

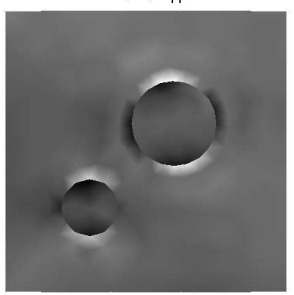

$c^{2}(10) C_{11}^{2}$

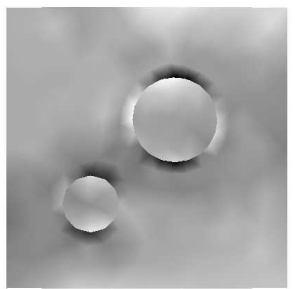

$c^{3}(10) c_{11}^{3}$

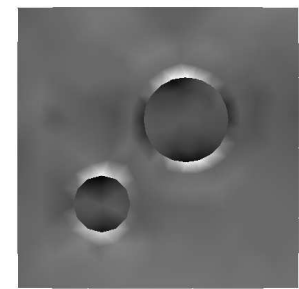

Fig. 32. Radial functions $\left\{c^{k}(t) \mathbf{C}^{k}(\underline{M})\right\}_{k=1}^{3}$ in Cell 1 at time $t=10 \mathrm{~s}$ Figure 33 shows the time functions $\left\{c^{k}(t)\right\}_{k=1}^{3}$.

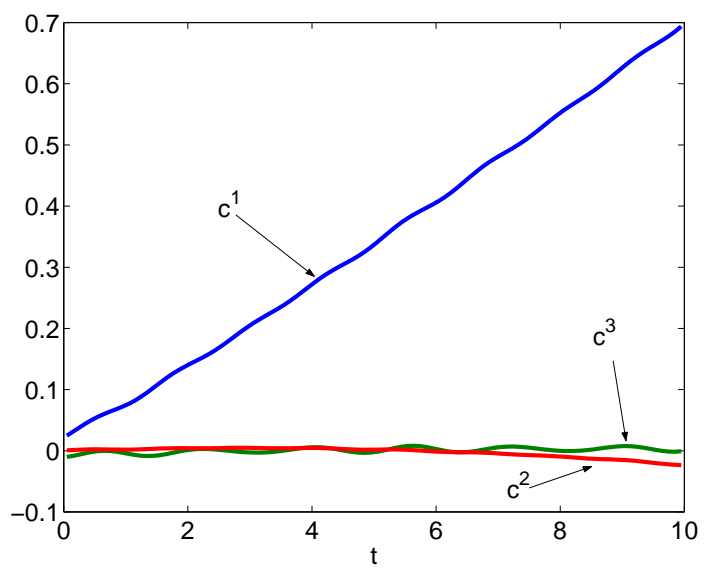

Fig. 33. Time functions $c^{k}(t)$ for Cell 1

We note that a single radial function represents the solution quite well. Table 2 shows the evolution of the error between the solution $(\boldsymbol{\sigma}, \underline{\mathrm{F}}, \boldsymbol{\varepsilon}, \underline{\mathrm{W}})$ in Cell 1 and the radial loading approximation with the number of radial functions.

Table 2

Relative error $\operatorname{err}_{\text {sta }}$ (reps. $\operatorname{err}_{k i n}$ ) between the approximation $\sum_{k=1}^{m} c^{k}\left(\mathbf{C}^{k}, \underline{G}^{k}\right)$ (reps. $\sum_{k=1}^{m} e^{k}\left(\mathbf{E}^{k}, \underline{\mathrm{Z}}^{k}\right)$ ) and $\boldsymbol{\sigma}$ (reps. $\boldsymbol{\varepsilon}$ ) on Cell 1 vs. the number of radial functions

\begin{tabular}{|c|c|c|}
\hline$m$ & $e r r_{\text {sta }}$ & err $_{\text {kin }}$ \\
\hline 1 & 0.0472 & 0.0121 \\
\hline 2 & 0.0450 & 0.0025 \\
\hline 3 & 0.0425 & 0.0009 \\
\hline 4 & 0.0279 & 0.0008 \\
\hline 5 & 0.0095 & 0.0006 \\
\hline
\end{tabular}

In conclusion, only a very small number of functions is needed to approximate 
the solution defined over the entire space-time domain and, therefore, the cost of the linear stage, which is the most costly stage, can be reduced drastically.

\section{Conclusion}

The improved method presented here led to significant performance gains in the resolution of the linear stage. The first improvement was the introduction, for composite structures, of a third scale and the use of an approximation technique for the macro problem: this approximation is based on an analogy between the macro homogenized structure and a Cosserat medium. The second improvement was the introduction of the radial loading approximation to solve the micro problems. This strategy extends the approximation classically used in the LATIN method for quasi-static problems [14]. The gain obtained by these two improvements becomes significant as one has to deal with a large number of composite cells and needs to calculate a detailed solution in the time domain. The techniques described here can be used only with global methods in space and in time, which excludes classical incremental methods.

\section{References}

[1] T. Belytschko, P. Smolinski, W. K. Liu, Stability of multi-time step partitioned integrators for the first-order finite element systems, Computer Methods in Applied Mechanics and Engineering, 49:281-297, 1985

[2] A. Benssoussan, J.-L. Lions, G. Papanicolaou, Asymptotic Analysis for Periodic Structures, North-Holland, 1978

[3] Bottasso C.L. , Multiscale temporal integration, Computer Methods in Applied Mechanics and Engineering, 191:2815-2830, 2002

[4] F. Devries, H. Dumontet, G. Duvaut, F. Léné, Homogenization and damage for composite structures, Interanational Journal for Numerical Methods in Engineering, 27:285-298, 1989

[5] D. Dureisseix, P. Ladevèze, D. Néron and B. A. Schrefler, A multi-time-scale strategy for multiphysics problems: application to poroelasticity, submitted to Journal of Multiscale Computational Engineering

[6] K. Eriksson, D. Estep, P.Hansbo, C. Johnson, Computational Differential Equations, Cambridge, 1996

[7] C. Farhat, M. Chandesris, Time-Decomposed Parallel Time-Integrators - Part I: Theory and Feasibility Studies for Fluid, Structure, and Fluid-Structure Applications, University of Colorado at Boulder, CU-CAS-02-10, 2002 
[8] F. Feyel, J.-L. Chaboche, $F E^{2}$ multiscale approach for modelling the elastoviscoplastic behaviour of long fiber $\mathrm{SiC} / \mathrm{Ti}$ composite materials, Computer Methods in Applied Mechanics and Engineering, 183:417-455, 2000

[9] J. Fish, K. Sheck, M. Pandheeradi, M.S. Shepard, Computational plasticity for composite structures based on mathematical homogenization : Theory and practice, Computer Methods in Applied Mechanics and Engineering, 148:53-73, 1997

[10] G.A. Francfort, Homogenization and linear thermoelasticity, SIAM Journal of Mathematical Analysis 14:696-708, 1983

[11] V. Kouznetsova, M. G. D. Geers, W. A. M. Brekelmans, Multi-scale constitutive modelling of heterogeneous materials with a gradient-enhanced computational homogenization scheme, Interanational Journal for Numerical Methods in Engineering, 54:1235-1260, 2002

[12] A. Gravouil, A. Combescure, Multi-time-step explicit implicit method for nonlinear structural dynamics, Interanational Journal for Numerical Methods in Engineering, 50:199-225, 2001

[13] T. Guennouni, Sur une méthode de calcul de structures soumises à des chargements cycliques : l'homogénéisation en temps, Mathematical Modelling and Numerical Analysis, 22(3):417-455, 1988

[14] P. Ladevèze, Nonlinear Computational Structural Mechanics - New Approaches and Non-Incremental Methods of Calculation, Springer Verlag, 1999

[15] P. Ladevèze, O. Loiseau, D. Dureisseix, A micro-macro and parallel computational strategy for highly heterogeneous structures, International Journal for Numerical Methods in Engineering, 52:121-138, 2001

[16] P. Ladevèze, A. Nouy, O. Loiseau, A multiscale computational approach for contact problems, Computer Methods in Applied Mechanics and Engineering, 191:4869-4891, 2002

[17] P. Ladevèze, A. Nouy, A multiscale computational method with time and space homogenization, C. R. Mécanique, 330:1-7, 2002

[18] P. Ladevèze, Multiscale Modelling and Computational Strategies for Composites, Proceedings of the Fifth World Congress on Computational Mechanics (WCCM V), July 7-12, 2002, Vienna, Austria, Editors: Mang, H.A.; Rammerstorfer, F.G.; Eberhardsteiner, J., Publisher: Vienna University of Technology, Austria, ISBN 3-9501554-0-6

[19] J.T. Oden, K. Vemaganti, N. Moes, Hierarchical modelling of heterogeneous solids, Computer Methods in Applied Mechanics and Engineering, 172:2-25, 1999

[20] E. Sanchez-Palencia, Comportement local et macroscopique d'un type de mileux physiques hétérogènes, International Journal for Engineering Science, 12:231251,1974 
[21] Smit R.J.M., Brekelmans W.A.M., Meijer H.E.H., Prediction of the mechanical behaviour of non-linear heterogeneous systems by multi-level finite element modelling, Computer Methods in Applied Mechanics and Engineering, 155:181192,1998

[22] Zohdi T.I., Oden J.T., Rodin G.J., Hierarchical modeling of heterogeneous bodies, Computer Methods in Applied Mechanics and Engineering, 138:273-298, 1996 